TRIHALOMETHANE AND NONPURGEABLE TOTAL ORGANIC-HALIDE FORMATION POTENTIALS FOR THE MISSISSIPPI RIVER AND SOME OF ITS TRIBUTARIES, SEPTEMBER-OCTOBER 1991

By R.E. Rathbun and L.M. Bishop

U.S. GEOLOGICAL SURVEY

Open-File Report 94-36 


\section{U.S. DEPARTMENT OF THE INTERIOR \\ BRUCE BABBITT, Secretary \\ U.S. GEOLOGICAL SURVEY \\ Gordon P. Eaton, Director}

The use of trade, product, industry, or firm names is for descriptive purposes only and does not imply endorsement by the U.S. Government.

For additional information write to:

Chief, Branch of Regional Research

U.S. Geological Survey

Box 25046, MS 418

Denver Federal Center

Denver, CO 80225
Copies of this report can be purchased from:

U.S. Geological Survey

Earth Science Information Center

Open-File Reports Section

Box 25286, MS 517

Denver Federal Center

Denver, CO 80225 


\section{CONTENTS}

Abstract

Introduction

Purpose and scope

Acknowledgments

Procedure for determination of trihalomethane and nonpurgeable total organic-halide

formation potentials

Sampling procedure

Formation-potential experiments

Analysis of trihalomethane samples

Analysis of nonpurgeable total organic-halide samples

Determination of $\mathrm{pH}$

Determination of dissolved organic-carbon and bromide concentrations

Determination of specific conductances

Determination of ultraviolet absorbances

Results of sample collection and formation-potential experiments

Trihalomethane and nonpurgeable total organic-halide formation potentials

Ancillary data

Values of $\mathrm{pH}$ as a function of reaction time

Values of $\mathrm{pH}$ at the end of the trihalomethane formation-potential experiments

Dissolved organic-carbon and bromide concentrations and specific conductances

Ultraviolet absorbances

Error analysis

References cited

\section{FIGURES}

1. Map showing location of sampling sites in the Mississippi River basin

2. Graph showing trihalomethane, nonpurgeable total organic-halide, and dissolvedorganic carbon concentrations as a function of cumulative river kilometers upstream from Head of Passes, La.; initial $\mathrm{pH}$ of 10.00 and initial free-chlorine concentration of 30.0 milligrams per liter

3. Graph showing trihalomethane and nonpurgeable total organic-halide concentrations as a function of reaction time for several sampling sites along the Mississippi River; initial $\mathrm{pH}$ of 7.50 and initial free-chlorine concentration of 30.0 milligrams per liter 


\section{TABLES}

Page

1. General location and cumulative river kilometers upstream from Head of Passes, La., for the sampling sites, dates and times of sampling, and water discharges--_- 3

2-15. Concentrations of the four trihalomethane compounds and the total trihalomethane and nonpurgeable total organic-halide concentrations for a reaction time of 168.0 hours as a function of initial $\mathrm{pH}$ and initial free-chlorine concentration:

2. Mississippi River at Minneapolis, Minn., October 7, 1991 -

3. Mississippi River at LaCrosse, Wis., October 4, 1991 -13

4. Mississippi River at Dubuque, Iowa, October 3, 1991

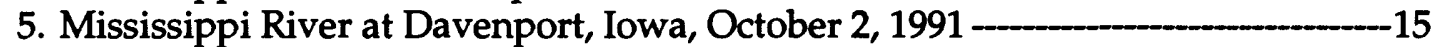

6. Mississippi River at Quincy, Ill., October 1, 1991 -16

7. Missouri River 1.6 kilometers upstream from confluence with Mississippi River, September 30, 1991 $-17$

8. Mississippi River at St. Louis, Mo., September 30, $1991 \ldots$

9. Mississippi River at Cairo, Ill., September 30, 1991 ________________

10. Ohio River 1.6 kilometers upstream from confluence with Mississippi River, September 29, 1991 -

11. Mississippi River at Memphis, Tenn., September 28, $1991 \ldots$

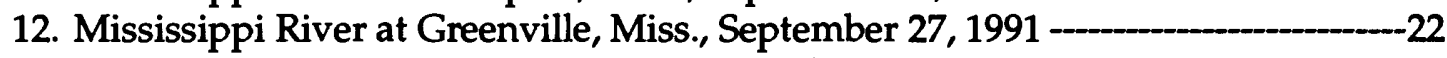

13. Mississippi River at Natchez, Miss., September 26, 1991

14. Mississippi River at Baton Rouge, La., September 26, 1991 -ב_____-_-24

15. Mississippi River at New Orleans, La., September 25, $1991 \ldots 25$

16-29. Concentrations of the four trihalomethane compounds and the total trihalomethane and nonpurgeable total organic-halide concentrations as a function of reaction time for an initial $\mathrm{pH}$ of 7.50 and an initial free-chlorine concentration of 30.0 milligrams per liter:

16. Mississippi River at Minneapolis, Minn., October 7, 1991 -_-_26

17. Mississippi River at LaCrosse, Wis., October 4, 1991 -26

18. Mississippi River at Dubuque, Iowa, October 3, 1991

19. Mississippi River at Davenport, Iowa, October 2, $1991 \ldots 27$

20. Mississippi River at Quincy, Ill., October 1, $1991 \ldots 28$

21. Missouri River 1.6 kilometers upstream from confluence with Mississippi River, September 30, 1991

22. Mississippi River at St. Louis, Mo., September 30, 1991 _-29

23. Mississippi River at Cairo, Ill., September 30, 1991 -._-29

24. Ohio River 1.6 kilometers upstream from confluence with Mississippi River, September 29, 1991 -

25. Mississippi River at Memphis, Tenn., September 28, 1991 -__-_-_-_30

26. Mississippi River at Greenville, Miss., September 27, 1991 _________-31

27. Mississippi River at Natchez, Miss., September 26, $1991 \ldots$

28. Mississippi River at Baton Rouge, La., September 26, 1991_-_______-32

29. Mississippi River at New Orleans, La., September 25, 1991 - 
30-43. Variation of $\mathrm{pH}$ with reaction time for different combinations of initial $\mathrm{pH}$ and initial free-chlorine concentration:

30. Mississippi River at Minneapolis, Minn., October 7, 1991 -

31. Mississippi River at LaCrosse, Wis., October 4, 1991 -

32. Mississippi River at Dubuque, Iowa, October 3, 1991_-35

33. Mississippi River at Davenport, Iowa, October 2, 1991 -36

34. Mississippi River at Quincy, Ill., October 1, 1991 -37

35. Missouri River 1.6 kilometers upstream from confluence with Mississippi River, September 30, 1991

36. Mississippi River at St. Louis, Mo., September 30, 1991 -39

37. Mississippi River at Cairo, Ill., September 30, 1991 - 40

38. Ohio River 1.6 kilometers upstream from confluence with Mississippi River, September 29, 1991

39. Mississippi River at Memphis, Tenn., September 28, 1991 -

40. Mississippi River at Greenville, Miss., September 27, 1991 -

41. Mississippi River at Natchez, Miss., September 26, 1991 -44

42. Mississippi River at Baton Rouge, La., September 26, 1991 - 45

43. Mississippi River at New Orleans, La., September 25, 1991 -_._-_6

44. Values of $\mathrm{pH}$ at the end of the trihalomethane formation-potential experiments ----48

45. Dissolved organic-carbon and bromide concentrations and specific conductances of the water samples

46. Ultraviolet absorbances of the water samples -

47. Minimum, maximum, and mean values of the coefficient of variation for analysis of duplicate trihalomethane and nonpurgeable total organic-halide samples $-52$ 


\section{CONVERSION FACTORS}

\begin{tabular}{lll} 
Multiply & By & To obtain \\
nanometer $(\mathrm{nm})$ & $3.937 \times 10^{-8}$ & inch \\
micrometer $(\mu \mathrm{m})$ & $3.937 \times 10^{-5}$ & inch \\
millimeter $(\mathrm{mm})$ & $3.937 \times 10^{-2}$ & inch \\
meter $(\mathrm{m})$ & $3.937 \times 10^{1}$ & inch \\
kilometer $(\mathrm{km})$ & 0.6214 & mile \\
microgram per liter $(\mu \mathrm{g} / \mathrm{L})$ & $6.243 \times 10^{-8}$ & pound per cubic foot \\
milligram per liter $(\mathrm{mg} / \mathrm{L})$ & $6.243 \times 10^{-5}$ & pound per cubic foot \\
microliter $(\mu \mathrm{L})$ & $3.531 \times 10^{-8}$ & cubic foot \\
milliliter $(\mathrm{mL})$ & $3.531 \times 10^{-5}$ & cubic foot \\
liter $(\mathrm{L})$ & $3.531 \times 10^{-2}$ & cubic foot \\
cubic meter per second $\left(\mathrm{m}^{3} / \mathrm{s}\right)$ & $3.531 \times 10^{1}$ & cubic foot per second \\
\hline
\end{tabular}

Temperature in degree Celsius $\left({ }^{\circ} \mathrm{C}\right)$ may be converted to degree Fahrenheit $\left({ }^{\circ} \mathrm{F}\right)$ using:

$$
{ }^{\circ} \mathrm{F}={ }^{\circ} \mathrm{C}(1.8)+32.0
$$




\title{
TRIHALOMETHANE AND NONPURGEABLE TOTAL ORGANIC- HALIDE FORMATION POTENTIALS FOR THE MISSISSIPPI RIVER AND SOME OF ITS TRIBUTARIES, SEPTEMBER-OCTOBER 1991
}

\author{
R.E. Rathbun and L.M. Bishop
}

\begin{abstract}
Trihalomethane and nonpurgeable total organic-halide formation potentials were determined for the Mississippi River and some of its tributaries from water samples collected between September 25, 1991, and October 7, 1991. Formation potentials were measured for 12 sites along the Mississippi River between Minneapolis, Minnesota, and New Orleans, Louisiana, and for the Missouri and Ohio Rivers at sites 1.6 kilometers upstream from their confluences with the Mississippi River. Formation potentials were determined as a function of initial $\mathrm{pH}$ and initial free-chlorine concentration for one reaction time of 168.0 hours at a temperature of 25 degrees Celsius. The formation potentials also were determined as a function of reaction time over a 168.0-hour period for an initial $\mathrm{pH}$ of 7.50 and an initial free-chlorine concentration of 30.0 milligrams per liter. Ancillary measurements include determinations of $\mathrm{pH}$ as a function of reaction time, $\mathrm{pH}$ values at the end of the formation-potential experiments, dissolved organiccarbon and bromide concentrations, ultraviolet absorbances, and specific conductances. All experiments except the reaction-time experiments were done in duplicate, thus permitting an analysis of the errors involved in determining the trihalomethane and nonpurgeable total organichalide formation potentials. Results of the formation-potential determinations, the ancillary measurements, and the error analysis are listed in tables.
\end{abstract}

\section{INTRODUCTION}

The Mississippi River begins in Lake Itasca in north-central Minnesota and drains into the Gulf of Mexico, 3,720 km downstream at a point in Louisiana called Head of Passes. The river flows through Minnesota and Louisiana and forms parts of the borders of Wisconsin, Iowa, Illinois, Missouri, Kentucky, Tennessee, Arkansas, and Mississippi. Numerous cities, towns, and villages in these States use the Mississippi River as a source of drinking water. Other population centers along the river might begin to use the Mississippi River as a source of drinking water as ground-water supplies are depleted and as the population increases.

To make water from the Mississippi River safe for drinking, the water first must be disinfected, usually with free chlorine, to eliminate the possibility of the transmission of water-borne diseases such as typhoid fever. Research (Rook, 1974; Bellar and others, 1974; Fleischacker and Randtke, 1983; and Johnson and Jensen, 1986) has indicated, however, that chlorination of natural waters results in the formation of various byproducts, some of which may be health hazards. Therefore, the potential for the formation of these byproducts must be considered when the Mississippi River is used as a source of drinking water.

These byproduct compounds are divided into two classes. The first class consists of the trihalomethane (THM) compounds, which are chlorinated and brominated derivatives of methane. The four compounds commonly included in this class are chloroform, bromodichloromethane, 
chlorodibromomethane, and bromoform. These compounds are volatile and can be determined by gas chromatography techniques. The second class consists of chlorinated and brominated compounds with molecular weights higher than those of the THM compounds. Compounds in this second class are more polar and generally are nonvolatile. They are more difficult to determine as individual compounds; consequently, they commonly are determined together as a bulk parameter called the total organic-halide (TOX) concentration. Because this measurement also includes the THM compounds, the samples usually are purged with an inert gas before analysis to remove the volatile THM compounds. This procedure results in the nonpurgeable total organic-halide (NPTOX) concentration.

The U.S. Geological Survey Mississippi River project is a multidisciplinary study of the waterquality characteristics of the river. Specific topics of study include the distribution of agricultural pesticides and herbicides, trace metals, and industrial organic chemicals among the water, sediment, and biotic phases of the river system and how these substances are transported by the river. Also of interest are the water-quality characteristics of the Mississippi River related to its use as a source of drinking water. Water characteristics affecting this use include constituents already present in the water as well as constituents that are formed when the water is chlorinated to make it safe for drinking. The objective of this report is to describe the results of the determination of disinfection byproducts that could be formed when water from the Mississippi River is chlorinated.

\section{Purpose and Scope}

This report presents the results of the determination of THM and NPTOX formation potentials for the Mississippi River and some of its tributaries. Formation potentials for the THM and NPTOX compounds are defined as the concentrations of these compounds that are formed when water samples are chlorinated at specific conditions of $\mathrm{pH}$, free-chlorine concentration, reaction time, and temperature. In this study, formation potentials were determined for water from 12 sites along the Mississippi River between Minneapolis, Minn., and New Orleans, La., and for water from the Missouri and Ohio Rivers at sites $1.6 \mathrm{~km}$ upstream from their confluences with the Mississippi River. Water samples were collected between September 25, 1991, and October 7, 1991. Formation potentials were determined as a function of initial $\mathrm{pH}$ and initial free-chlorine concentration for one reaction time of 168.0 hours at a temperature of $25^{\circ} \mathrm{C}$. Also, the formation potentials were determined as a function of reaction time over a 168.0-hour period for an initial $\mathrm{pH}$ of 7.50 and an initial free-chlorine concentration of $30.0 \mathrm{mg} / \mathrm{L}$.

\section{Acknowledgments}

Water samples upon which this work was based were collected as a part of the U.S. Geological Survey Mississippi River project under the direction of R.H. Meade. J.A. Moody provided logistical support. Craig LeBoeuf and George Collier piloted the research vessel ACADIANA, and Chuck Guidry was the cook. L.B. Barber II, D.A. Goolsby, Dick Martin, Harold Wiegner, and Charles Tabor assisted with the sampling. J.A. Moody collected the Minneapolis, Minn., sample. Appreciation is expressed to all who helped with this study. 


\section{PROCEDURE FOR DETERMINATION OF TRIHALOMETHANE AND NONPURGEABLE TOTAL ORGANIC-HALIDE FORMATION POTENTIALS}

\section{Sampling Procedure}

The 17-m research vessel ACADIANA, owned and operated by the Louisiana Universities Marine Consortium, was used for collecting samples. Grab samples of water were collected by dropping a stainless-steel bucket off the port side of the vessel. The bucket was rinsed once with river water before collecting the sample. Single samples usually were collected from the midpoint of the navigation channel. One exception was the Ohio River, where there was a question about whether the various tributaries flowing into the Ohio River would be mixed at the sampling location. Consequently, a composite sample of equal volumes was collected from points located at 0.1 , 0.5 , and 0.9 of the width measured from the left edge of the water.

On the Mississippi River, the sampling locations generally were slightly upstream from metropolitan areas. General locations in terms of the metropolitan area and specific locations in terms of cumulative river kilometers upstream from Head of Passes, La., for the sampling sites, dates and times of sample collection, and water discharges at the sampling times are listed in table 1. Location of the sampling sites is shown in figure 1.

Table 1.-General location and cumulative river kilometers upstream from Head of Passes, La., for the sampling sites, dates and times of sampling, and water discharges

$\left[\mathrm{m}^{3} / \mathrm{s}\right.$, cubic meters per second; $\mathrm{NQ}$, water discharge not available]

\begin{tabular}{lcccc}
\hline General location & $\begin{array}{c}\text { Cumulative } \\
\text { river } \\
\text { kilometers }\end{array}$ & Date & $\begin{array}{c}\text { Time } \\
\text { (hours) }\end{array}$ & $\begin{array}{c}\text { Water } \\
\text { discharge } \\
\left.\text { (m } \mathbf{m}^{3} / \mathbf{s}\right)\end{array}$ \\
\hline Minneapolis, Minn. & $2,915.2$ & $10-07-91$ & 1030 & 230 \\
LaCrosse, Wis. & $2,666.9$ & $10-04-91$ & 0151 & 940 \\
Dubuque, Iowa & $2,470.7$ & $10-03-91$ & 0904 & 1,300 \\
Davenport, Iowa & $2,325.2$ & $10-02-91$ & 2141 & 1,400 \\
Quincy, Ill. & $2,068.4$ & $10-01-91$ & 2107 & 2,000 \\
Missouri River ${ }^{2}$ & $1,850.8$ & $09-30-91$ & 2333 & 1,100 \\
St. Louis, Mo. & $1,825.1$ & $09-30-91$ & 2140 & 3,200 \\
Cairo, Ill. & $1,552.3$ & $09-30-91$ & 0039 & 3,700 \\
Ohio River ${ }^{2}$ & $1,536.5$ & $09-29-91$ & 2122 & 1,800 \\
Memphis, Tenn. & $1,194.1$ & $09-28-91$ & 1811 & 5,600 \\
Greenville, Miss. & 877.1 & $09-27-91$ & 1544 & 6,200 \\
Natchez, Miss. & 597.4 & $09-26-91$ & 1752 & 7,200 \\
Baton Rouge, La. & 386.9 & $09-26-91$ & 0241 & 4,500 \\
New Orleans, La. & 169.1 & $09-25-91$ & 0833 & NQ \\
\hline
\end{tabular}

${ }^{1}$ Discharge data from Moody and Meade (U.S. Geological Survey, written commun., 1993).

${ }^{2}$ Sampling site 1.6 kilometers upstream from confluence with Mississippi River. 


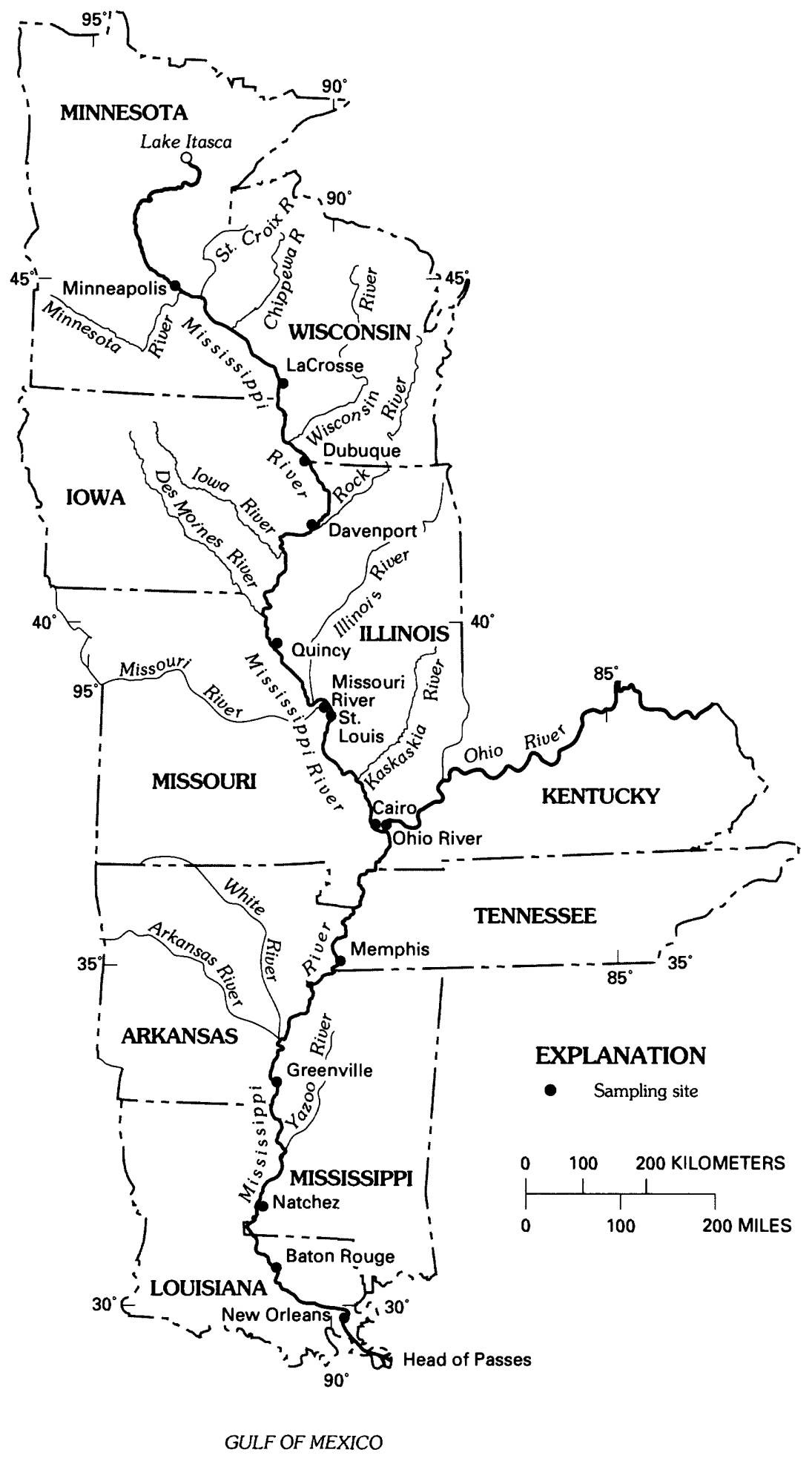

Figure 1.-Location of sampling sites in the Mississippi River basin. 
The water sample collected at each site was transferred from the bucket to a stainless-steel reservoir for pressure filtration. The water was filtered through low-extractable $0.45-\mu \mathrm{m}$ membrane filters that were prerinsed with about $2 \mathrm{~L}$ of high-purity water from a Barnstead Nanopure water system. The first $600 \mathrm{~mL}$ of sample water through the filter was discarded. The water collected at each site was filtered into six 1-L amber glass bottles with Teflon-lined caps. The sample bottles were chilled with ice until shipment to the laboratory in Arvada, Colo. Shipment was by overnight air freight in insulated coolers containing refrigerant cold packs. Water samples in the laboratory were stored at $4^{\circ} \mathrm{C}$ until the formation-potential experiments were done.

\section{Formation-Potential Experiments}

The formation potentials for the THM and NPTOX compounds were determined as a function of initial $\mathrm{pH}$ and initial free-chlorine concentration at $25^{\circ} \mathrm{C}$ for a reaction time of 168.0 hours. Initial $\mathrm{pH}$ values of 5.50,7.50, and 10.00 and initial free-chlorine concentrations of 15.0,30.0, and $50.0 \mathrm{mg} / \mathrm{L}$ were used, giving nine THM and nine NPTOX formation potentials for each water sample. These large initial free-chlorine concentrations were used to satisfy any initial chlorine demand of the water sample and to ensure a residual free-chlorine concentration at the end of the 168.0-hour reaction time. The 168.0-hour reaction time was used to cover the maximum possible time water might be in a distribution system before use. Formation potentials also were determined as a function of time during the 168.0-hour period for an initial $\mathrm{pH}$ of 7.50 and an initial free-chlorine concentration of $30.0 \mathrm{mg} / \mathrm{L}$.

The formation-potential experiments were done in $40-\mathrm{mL}$ amber glass vials with Teflon-faced septum caps. Initiation of an experiment consisted of adjusting the $\mathrm{pH}$ of the water sample to the desired initial $\mathrm{pH}$ using 0.1 normal and 1.0 normal sulfuric acid and sodium hydroxide. The desired initial free-chlorine concentration was obtained by adding the appropriate volume of a reagent-grade sodium hypochlorite solution. The free-chlorine concentration of this solution was determined prior to the start of each experiment by using an amperometric titration procedure. Blank samples of each water sample were taken before the addition of the sodium hypochlorite solution.

Sample vials were filled completely so that no head space existed. Vials were placed in an incubator at $25^{\circ} \mathrm{C}$. All 168.0-hour experiments were done with duplicate vials. At the end of 168.0 hours, the experiments were terminated by quenching the remaining free chlorine with a sodium thiosulfate solution for the THM experiments and sodium sulfite crystals for the NPTOX experiments. For the reaction-time experiments, samples were quenched at approximate times of $0,2,6,12,24,48,72,120$, and 168.0 hours.

Before quenching the NPTOX samples, $5.0 \mathrm{~mL}$ of sample was removed from each of the vials and used to estimate the residual free-chlorine concentrations. The concentration of residual freechlorine was estimated using a Hach chlorine test kit, which has a range from 0 to $3.5 \mathrm{mg} / \mathrm{L}$ with $0.1-\mathrm{mg} / \mathrm{L}$ divisions. After acidification to a $\mathrm{pH}$ of about 2 with nitric acid, the NPTOX samples were purged for 10 minutes with ultrahigh-purity nitrogen gas to remove the THM compounds. Samples were stored in an incubator at $4^{\circ} \mathrm{C}$ until analysis. 


\section{Analysis of Trihalomethane Samples}

Concentrations of the THM compounds in the samples were determined with a solventextraction procedure (Federal Register, 1979) with pentane as the solvent. The procedure was modified slightly in that the sample extractions were done directly in the sample vials to avoid having to transfer a sample containing volatile constituents. The procedure consisted of removing and discarding $4.5 \mathrm{~mL}$ of sample, adding $4.0 \mathrm{~mL}$ of pentane, and shaking the mixture for 1.5 minutes. The Burdick and Jackson brand of pentane especially formulated for THM analyses was used. The vials containing the water and pentane layers were stored upside down at $4^{\circ} \mathrm{C}$ until analysis. Storing the samples upside down precluded any loss of the pentane or the THM compounds from the sample vial during storage.

The pentane extracts were analyzed with a Hewlett-Packard 5880-A gas chromatograph equipped with an electron-capture detector. A 12-m Hewlett-Packard, cross-linked, methyl silicone-gum capillary column was used with the purged splitless technique. Retention times on this column were $0.92,1.42,2.49$, and 4.77 minutes for chloroform, bromodichloromethane, chlorodibromomethane, and bromoform, respectively.

Volumes of the $40-\mathrm{mL}$ sample vials were not precisely $40.0 \mathrm{~mL}$ and were not consistent. To adjust for variations in the vial volumes, the vials were weighed at each step of the extraction process, beginning with the empty vial and ending with the final sample/pentane step. These weights permitted determination of the exact water/pentane volume ratio for each sample. The peak areas for each sample were adjusted from the volume ratio of that sample to the average volume ratio for the standard samples for that particular run. This procedure resulted in all samples and standards being adjusted to a single water/pentane ratio.

Standard samples of known concentrations of each of the four THM compounds were prepared according to an established procedure (Federal Register, 1979). The procedure consists of adding each of the four THM compounds to methanol in a $10-\mathrm{mL}$ volumetric flask. The amount added was determined approximately by volume and precisely by weighing on a five-place analytical balance. Two additional primary standards were prepared by serial dilution of this standard using gas-tight syringes. Secondary standards covering the expected range of sample concentrations were prepared by injecting microliter quantities of the primary standards into river water contained in 40-mL septum vials. River water was used to prepare the standards to eliminate any possible effect of the water chemistry on the efficiency of the extraction procedure. These secondary standards were treated exactly as the samples from the experiments except that sodium hypochlorite was not added to the standards. A new set of standards was prepared for each river-water sample.

A large number of standards was necessary because the response of the electron-capture detector was somewhat nonlinear. Also, the standards had to cover a wide range of concentrations so that the peak areas of each of the four THM compounds in every sample were bracketed by the peak areas of the standards. The number of standards ranged from 16 for the Minneapolis sample to 11 for the New Orleans sample. These standards were analyzed twice each day, resulting in 42 to 53 percent of the samples analyzed each day being standards. 


\section{Analysis of Nonpurgeable Total Organlc-Halide Samples}

Concentrations of the NPTOX compounds in the samples were determined using a Dohrmann DX-20A TOX analyzer with an AD-3 adsorption module. This procedure involved adsorption of the organic compounds from the water sample onto granular activated carbon packed in glass columns. Two of these columns were used in series for each sample. Following adsorption of the sample, the contents of the columns were washed with $2 \mathrm{~mL}$ of a 0.08 normal potassium nitrate solution to remove inorganic halides. The granular activated carbon then was removed from the columns and burned in a furnace at $800^{\circ} \mathrm{C}$ where the chlorinated and brominated compounds were converted to an equivalent amount of halide ions. These ions were titrated in a microcoulometric cell, resulting in the determination of the mass of NPTOX compounds in the original sample. The NPTOX concentration was computed from this mass and the volume of sample sorbed in the adsorption step.

The high concentrations of NPTOX compounds resulting from the formation-potential experiments required dilution of the samples to get the concentration within the response range of the instrument. Samples were diluted with high-purity water from a Barnstead Nanopure system. A blank determination for this dilution water was done each day samples were run. Usually, $30 \mathrm{~mL}$ of mixture was prepared, with the amount of sample ranging from 5 to $25 \mathrm{~mL}$, depending on the expected concentration. All aliquots were measured with Class A pipets, and $25 \mathrm{~mL}$ of this $30-\mathrm{mL}$ sample was forced through the glass columns containing the granular activated carbon using ultrahigh-purity nitrogen gas.

Operation of the microcoulometric cell was verified at the beginning of each day by injections of a sodium chloride standard directly into the electrolyte of the cell. These verifications also were done any time during the day when it became necessary to change the cell electrolyte. Recovery of the instrument was checked at the beginning of each day by injections of a trichlorophenol standard onto granular activated-carbon ash in the instrument boat and subsequent analysis of the trichlorophenol standard. These recovery checks also were repeated any time during the day when it became necessary to change the cell electrolyte and on any other occasion when operation of the instrument might be questionable. If results of these checks deviated by more than \pm 10 percent from the expected values, steps were taken to improve the performance of the instrument by changing the cell electrolyte and the inlet and exit tube liners and by cleaning the hatch area.

\section{Determination of $\mathrm{pH}$}

Initial $\mathrm{pH}$ values of the water samples were $5.50,7.50$, and 10.00 . Because these water samples were not buffered, the $\mathrm{pH}$ of the 5.50 and 7.50 water samples increased, and the $\mathrm{pH}$ of the 10.00 water samples decreased slightly when the basic sodium hypochlorite solution was added to provide the free-chlorine concentration. To determine the variation of the $\mathrm{pH}$ with time, the $\mathrm{pH}$ values of each of the nine combinations of initial $\mathrm{pH}$ and initial free-chlorine concentration were determined at reaction times of approximately $0,24,48,72,120,144$, and 168 hours.

Measurements were made using an Orion Model $811 \mathrm{pH}$ meter with a 91-02 research-grade $\mathrm{pH}$ probe. These samples were prepared at the same time the vials for the formation-potential experiments were filled. Amber glass bottles having Teflon-lined caps, a volume of $70 \mathrm{~mL}$, and a mouth wide enough to accommodate the $\mathrm{pH}$ and temperature probes of the instrument were used for this purpose. The $\mathrm{pH}$ meter was calibrated each day before the measurements were done. A two-point calibration using buffers of 6.86 and 10.00 bracketed all the experimental $\mathrm{pH}$ values. 
The $\mathrm{pH}$ values of the THM formation-potential experiments also were determined after completion of the gas chromatographic analyses. The pentane was evaporated, and the $\mathrm{pH}$ of the remaining water was determined for each of the nine samples.

\section{Determination of Dissolved Organic-Carbon and Bromide Concentrations}

Dissolved organic-carbon (DOC) and bromide concentrations of the water samples were determined at the U.S. Geological Survey National Water Quality Laboratory in Arvada, Colo. DOC concentration was determined using the wet-oxidation method (Wershaw and others, 1987). Duplicate samples were analyzed for all samples. Bromide concentrations were determined using segmented-flow automated colorimetry (Fishman and Friedman, 1989).

\section{Determination of Specific Conductances}

Specific conductances of the sample waters were measured using a Yellow Springs Instrument Company model 32 conductance meter. Temperatures of the water samples were adjusted to $25.0^{\circ} \mathrm{C}$ so that no temperature corrections were necessary.

\section{Determination of Ultraviolet Absorbances}

The ultraviolet (UV) absorbance of natural waters has been used as a predictor of the DOC concentration. Various UV wavelengths have been used (Buffle and others, 1978; Oliver and Thurman, 1981; Edzwald and others, 1985; Ceraso, 1987; Chadick and Amy, 1987; Moore, 1987). Therefore, UV absorbances of the water samples were determined at wavelengths of 254, 280,330, and $400 \mathrm{~nm}$. A Spectronics model 2000 spectrophotometer with a $50-\mathrm{mm}$ cell was used for these determinations. Absorbances were measured for both the $\mathrm{pH}$-adjusted water samples and the natural water samples.

\section{RESULTS OF SAMPLE COLLECTION AND FORMATION-POTENTIAL EXPERIMENTS}

\section{Trihalomethane and Nonpurgeable Total Organic-Halide Formation Potentials}

The potentials for formation of THM and NPTOX compounds when the water samples were treated with free chlorine are expressed as the concentrations of these compounds formed in the experiments. Two types of results were obtained. The first type consisted of concentrations of the THM and NPTOX compounds formed during a reaction time of 168.0 hours for nine combinations of initial $\mathrm{pH}$ and initial free-chlorine concentration. The second type consisted of concentrations of the THM and NPTOX compounds formed as a function of time during the reaction time of 168.0 hours for an initial $\mathrm{pH}$ of 7.50 and an initial free-chlorine concentration of $30.0 \mathrm{mg} / \mathrm{L}$. Typical results of the first type (fig. 2) show THM and NPTOX concentrations as a function of cumulative river kilometers upstream from Head of Passes, La., for an initial pH of 10.00 and an initial free-chlorine concentration of $30.0 \mathrm{mg} / \mathrm{L}$. The DOC concentrations also are shown in figure 2. Typical results of the second type (fig. 3) show THM and NPTOX concentrations as a function of reaction time for several sampling sites along the Mississippi River. 


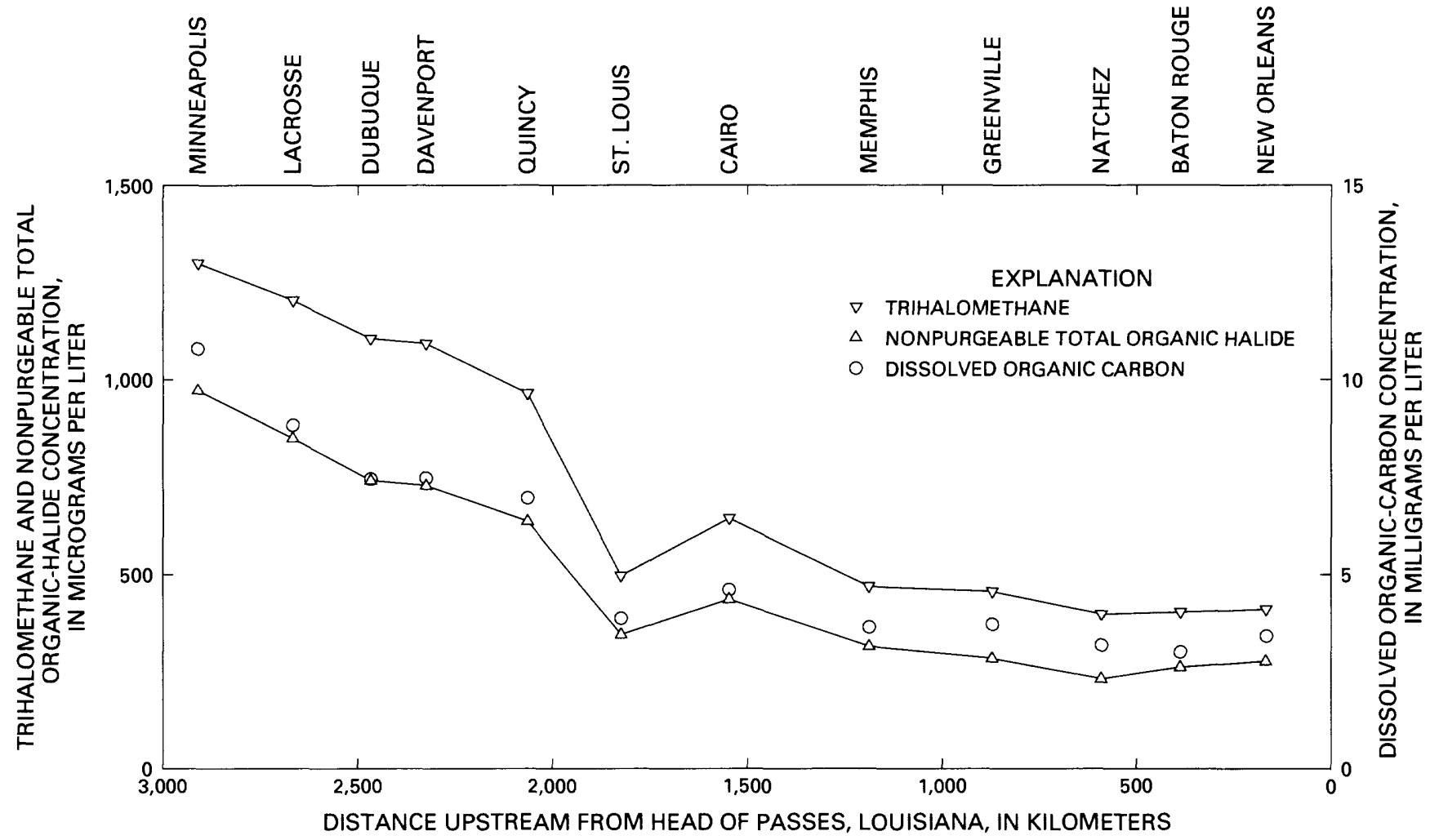

Figure 2.--Trihalomethane, nonpurgeable total organic-halide, and dissolved organic-carbon concentrations as a function of cumulative river kilometers upstream from Head of Passes, La.; initial $\mathrm{pH}$ of $\mathbf{1 0 . 0 0}$ and initial free-chlorine concentration of $\mathbf{3 0 . 0}$ milligrams per liter. 

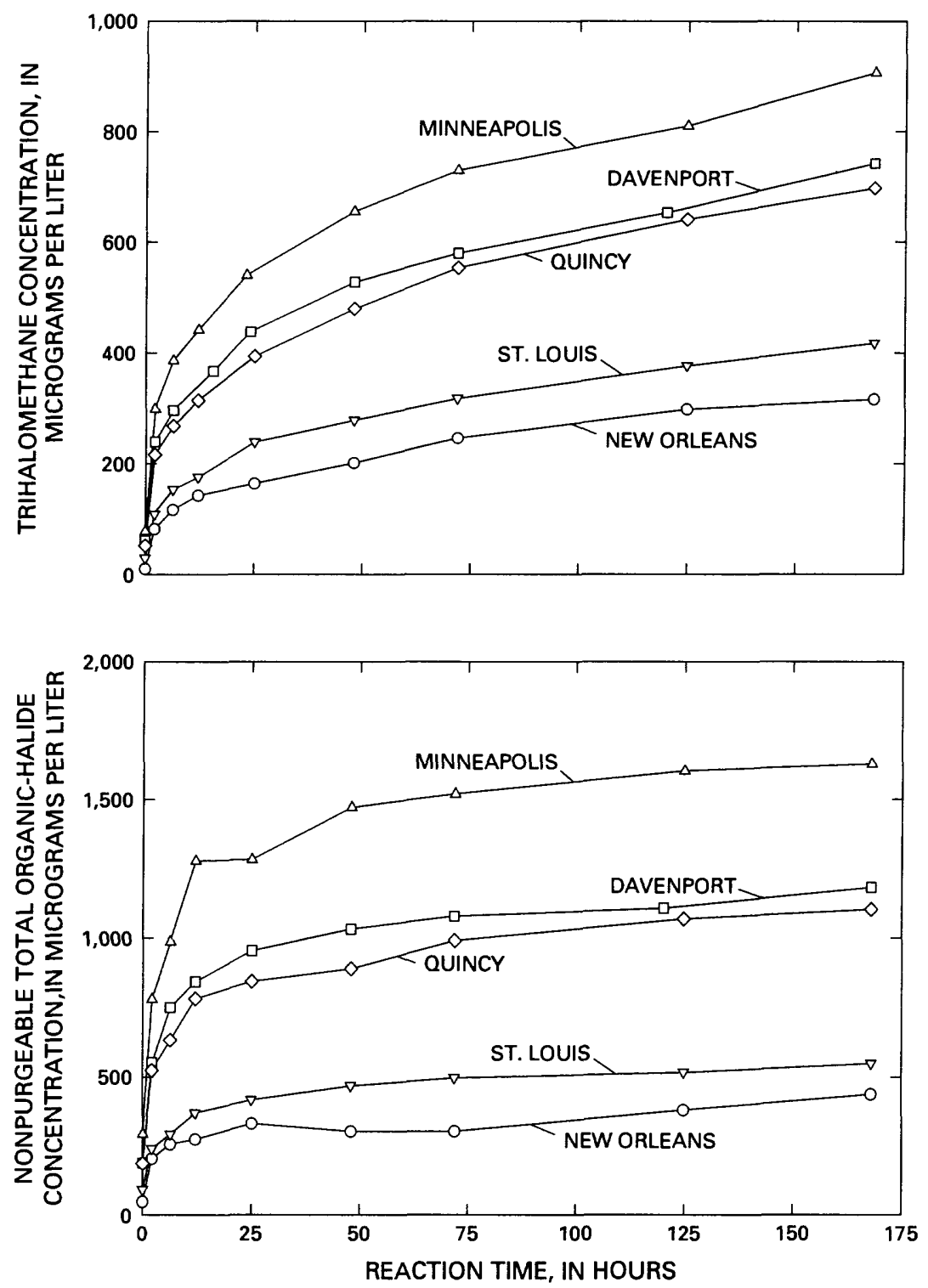

Figure 3.--Trihalomethane and nonpurgeable total organichalide concentrations as a function of reaction time for several sampling sites along the Mississippi River; initial pH of 7.50 and initial free-chlorine concentration of $\mathbf{3 0 . 0}$ milligrams per liter. 
All concentrations were corrected for blank contributions. The blank corrections for chloroform ranged from 0.15 to $0.97 \mu \mathrm{g} / \mathrm{L}$ and averaged $0.47 \mu \mathrm{g} / \mathrm{L}$ for 42 determinations. Average blank corrections for the other three THM compounds were less than $0.02 \mu \mathrm{g} / \mathrm{L}$. Blank corrections for the NPTOX concentrations ranged from 6.12 to $29.1 \mu \mathrm{g} / \mathrm{L}$ and averaged $17.1 \mu \mathrm{g} / \mathrm{L}$ for 36 determinations for samples from the Mississippi River. Corrections for the Missouri River water sample ranged from 4.85 to $8.05 \mu \mathrm{g} / \mathrm{L}$ and averaged $6.38 \mu \mathrm{g} / \mathrm{L}$. Corrections for the Ohio River water sample ranged from 15.6 to $18.8 \mu \mathrm{g} / \mathrm{L}$ and averaged $17.4 \mu \mathrm{g} / \mathrm{L}$.

The three chlorinated THM compounds (chloroform, bromodichloromethane, and chlorodibromomethane) were detected in all samples. Bromoform was not detected in 36 percent of the samples. The detection limit for bromoform for the analytical procedure used in this study was estimated to be about $0.01 \mu \mathrm{g} / \mathrm{L}$.

For the first type of results, duplicate experiments were done for all nine combinations of initial $\mathrm{pH}$ and initial free-chlorine concentration for each water sample. Some of the vials for the Minneapolis, LaCrosse, and Dubuque water samples had no detectable free-chlorine concentrations at the end of the reaction time. The limit of detection of the procedure used for these freechlorine measurements was $0.1 \mathrm{mg} / \mathrm{L}$. Concentrations for these samples are flagged with an asterisk in tables $2-4$ to indicate that these concentrations could have been affected by the lack of a free-chlorine residual at the end of the experiments.

For the second type of results, duplicate experiments were done for a reaction time of 168.0 hours and single experiments were done for all other reaction times. The 0.00 -hour reaction-time samples were samples taken and quenched immediately after addition of the sodium hypochlorite solution to the water sample. The time required to add the sodium hypochlorite solution to the water sample in a separatory funnel, to mix the contents, to fill the 0.00 -hour time vials already containing the quenching agent, and to shake these vials ranged from 30 to 45 seconds.

The first type of results consisting of concentrations of the four THM compounds and the total THM and NPTOX concentrations for a reaction time of 168.0 hours and nine combinations of initial $\mathrm{pH}$ and free-chlorine concentration are listed in tables 2-15. The second type of results consisting of concentrations of the four THM compounds and the total THM and NPTOX concentrations as a function of reaction time for an initial $\mathrm{pH}$ of 7.50 and an initial free-chlorine concentration of $30.0 \mathrm{mg} / \mathrm{L}$ are listed in tables 16-29. The concentrations for the 168.0-hour reaction time for an initial $\mathrm{pH}$ of 7.50 and an initial free-chlorine concentration of $30.0 \mathrm{mg} / \mathrm{L}$ are parts of both the first and second types of results. Therefore, these concentrations are presented in both tables 2-15 and tables 16-29. 
Table 2.-Concentrations of the four trihalomethane compounds and the total trihalomethane and nonpurgeable total organic-halide concentrations for a reaction time of $\mathbf{1 6 8 . 0}$ hours as a function of initial $\mathrm{pH}$ and initial free-chlorine concentration, Mississippi River at Minneapolis, Minn., October 7, 1991

$[\mu \mathrm{g} / \mathrm{L}$, microgram per liter; $\mathrm{CL}$, free-chlorine concentration; $\mathrm{mg} / \mathrm{L}$, milligram per liter; $\mathrm{CHCl}_{3}$, chloroform; $\mathrm{BrCHCl}_{2}$, bromodichloromethane; $\mathrm{ClCHBr}_{2}$, chlorodibromomethane; $\mathrm{CHBr}_{3}$, bromoform; THM, trihalomethane; NPTOX, nonpurgeable total organic halide; ND, not detected]

\begin{tabular}{|c|c|c|c|c|c|c|c|}
\hline \multirow{2}{*}{ Initial pH } & \multirow{2}{*}{$\begin{array}{c}\text { Initial CL } \\
\text { (mg/L) }\end{array}$} & \multicolumn{6}{|c|}{$\begin{array}{c}\text { Concentration } \\
(\mu g / L)\end{array}$} \\
\hline & & $\mathrm{CHCl}_{3}$ & $\mathrm{BrCHCl}_{2}$ & $\mathrm{ClCHBr}_{2}$ & $\mathrm{CHBr}_{3}$ & $\begin{array}{l}\text { Total } \\
\text { THM }\end{array}$ & NPTOX \\
\hline 5.50 & 15.0 & $425^{*}$ & $22.5^{*}$ & $0.33^{*}$ & ND* & $448^{*}$ & $1,250^{*}$ \\
\hline 5.50 & 15.0 & $426^{*}$ & $22.1^{*}$ & $.31^{*}$ & ND* & $448^{*}$ & $1,330^{*}$ \\
\hline 5.50 & 30.0 & 603 & 23.4 & .31 & ND & 627 & 1,830 \\
\hline 5.50 & 30.0 & 606 & 22.9 & .44 & ND & 629 & 1,960 \\
\hline 5.50 & 50.0 & 771 & 23.8 & .33 & ND & 795 & 2,210 \\
\hline 5.50 & 50.0 & 750 & 22.9 & .31 & ND & 773 & 2,290 \\
\hline 7.50 & 15.0 & $585^{*}$ & $25.6^{*}$ & $.45^{*}$ & ND* & $611^{*}$ & $1,040^{*}$ \\
\hline 7.50 & 15.0 & $592 *$ & $26.4^{*}$ & $.45 *$ & ND* & 619* & $1,020^{*}$ \\
\hline 7.50 & 30.0 & 873 & 31.1 & .47 & ND & 905 & 1,640 \\
\hline 7.50 & 30.0 & 883 & 31.0 & .47 & ND & 914 & 1,620 \\
\hline 7.50 & 50.0 & 1,150 & 30.8 & .50 & ND & 1,180 & 1,740 \\
\hline 7.50 & 50.0 & 1,120 & 28.2 & .45 & ND & 1,150 & 1,740 \\
\hline 10.00 & 15.0 & $856^{*}$ & $23.4^{*}$ & $.50^{*}$ & ND* & $880^{*}$ & $768 *$ \\
\hline 10.00 & 15.0 & $873^{*}$ & $23.7^{*}$ & $.53^{*}$ & ND* & $897^{*}$ & $780^{*}$ \\
\hline 10.00 & 30.0 & 1,300 & 28.6 & .58 & ND & 1,330 & 981 \\
\hline 10.00 & 30.0 & 1,290 & 28.7 & .61 & ND & 1,320 & 1,020 \\
\hline 10.00 & 50.0 & 1,500 & 30.6 & .60 & ND & 1,530 & 1,020 \\
\hline 10.00 & 50.0 & 1,510 & 30.5 & .59 & ND & 1,540 & 1,180 \\
\hline
\end{tabular}

${ }^{*}$ Free-chlorine concentration less than the detection limit at reaction time of 168.0 hours. 
Table 3.-Concentrations of the four trihalomethane compounds and the total trihalomethane and nonpurgeable total organic-halide concentrations for a reaction time of 168.0 hours as a function of initial pH and initial free-chlorine concentration, Mississippi River at LaCrosse, Wis., October 4, 1991

$[\mu \mathrm{g} / \mathrm{L}$, microgram per liter; $\mathrm{CL}$, free-chlorine concentration; $\mathrm{mg} / \mathrm{L}$, milligram per liter; $\mathrm{CHCl}_{3}$, chloroform; $\mathrm{BrCHCl}_{2}$, bromodichloromethane; $\mathrm{ClCHBr}$, chlorodibromomethane; $\mathrm{CHBr}_{3}$, bromoform; THM, trihalomethane; NPTOX, nonpurgeable total organic halide; ND, not detected]

\begin{tabular}{|c|c|c|c|c|c|c|c|}
\hline \multirow{2}{*}{ Initial pH } & \multirow{2}{*}{$\begin{array}{c}\text { Initial CL } \\
\text { (mg/L) }\end{array}$} & \multicolumn{6}{|c|}{$\begin{array}{c}\text { Concentration } \\
(\mu g / L)\end{array}$} \\
\hline & & $\mathrm{CHCl}_{3}$ & $\mathrm{BrCHCl}_{2}$ & $\mathrm{ClCHBr}_{2}$ & $\mathrm{CHBr}_{3}$ & $\begin{array}{l}\text { Total } \\
\text { THM }\end{array}$ & NPTOX \\
\hline 5.50 & 15.0 & 447 & 20.4 & 0.33 & ND & 468 & 1,310 \\
\hline 5.50 & 15.0 & 436 & 20.3 & .59 & ND & 457 & 1,320 \\
\hline 5.50 & 30.0 & 554 & 19.4 & .36 & ND & 574 & 1,660 \\
\hline 5.50 & 30.0 & 556 & 20.0 & .32 & ND & 576 & 1,630 \\
\hline 5.50 & 50.0 & 691 & 21.0 & .52 & ND & 713 & 1,880 \\
\hline 5.50 & 50.0 & 685 & 20.4 & .38 & ND & 706 & 1,930 \\
\hline 7.50 & 15.0 & $607^{*}$ & $23.7^{*}$ & $.49 *$ & ND* & $631^{*}$ & $1,020^{*}$ \\
\hline 7.50 & 15.0 & $609 *$ & $24.2^{*}$ & $.49 *$ & ND* & $634 *$ & 987* \\
\hline 7.50 & 30.0 & 822 & 28.2 & .52 & ND & 851 & 1,390 \\
\hline 7.50 & 30.0 & 818 & 27.8 & .51 & ND & 846 & 1,360 \\
\hline 7.50 & 50.0 & 1,030 & 26.7 & .52 & ND & 1,060 & 1,460 \\
\hline 7.50 & 50.0 & 995 & 26.4 & .51 & ND & 1,020 & 1,470 \\
\hline 10.00 & 15.0 & $926^{*}$ & $23.3^{*}$ & $.57^{*}$ & ND* & $950 *$ & $757 *$ \\
\hline 10.00 & 15.0 & $933^{*}$ & $23.6^{*}$ & $.69^{*}$ & ND* & $957 *$ & $700 *$ \\
\hline 10.00 & 30.0 & 1,200 & 28.0 & .60 & ND & 1,230 & 840 \\
\hline 10.00 & 30.0 & 1,170 & 26.5 & .55 & ND & 1,200 & 938 \\
\hline 10.00 & 50.0 & 1,340 & 28.1 & .57 & ND & 1,370 & 993 \\
\hline 10.00 & 50.0 & 1,350 & 28.4 & .56 & ND & 1,380 & 1,060 \\
\hline
\end{tabular}

*Free-chlorine concentration less than the detection limit at reaction time of 168.0 hours. 
Table 4.-Concentrations of the four trlhalomethane compounds and the total trihalomethane and nonpurgeable total organic-halide concentrations for a reaction time of 168.0 hours as a function of initial pH and initial free-chlorine concentration, Mississippi River at Dubuque, lowa, October 3, 1991

$[\mu \mathrm{g} / \mathrm{L}$, microgram per liter; $\mathrm{CL}$, free-chlorine concentration; $\mathrm{mg} / \mathrm{L}$, milligram per liter; $\mathrm{CHCl}_{3}$, chloroform; $\mathrm{BrCHCl}_{2}$, bromodichloromethane; $\mathrm{ClCHBr}$, chlorodibromomethane; $\mathrm{CHBr}_{3}$, bromoform; THM, trihalomethane; NPTOX, nonpurgeable total organic halide; ND, not detected]

\begin{tabular}{|c|c|c|c|c|c|c|c|}
\hline \multirow{2}{*}{ Initial pH } & \multirow{2}{*}{$\begin{array}{c}\text { Initial CL } \\
\text { (mg/L) }\end{array}$} & \multicolumn{6}{|c|}{$\begin{array}{c}\text { Concentration } \\
(\mu g / L)\end{array}$} \\
\hline & & $\mathrm{CHCl}_{3}$ & $\mathrm{BrCHCl}_{2}$ & $\mathrm{ClCHBr}_{2}$ & $\mathrm{CHBr}_{3}$ & $\begin{array}{l}\text { Total } \\
\text { THM }\end{array}$ & NPTOX \\
\hline 5.50 & 15.0 & 397 & 20.6 & 0.40 & ND & 418 & 1,130 \\
\hline 5.50 & 15.0 & 383 & 19.2 & .38 & ND & 403 & 1,180 \\
\hline 5.50 & 30.0 & 506 & 20.2 & .38 & ND & 527 & 1,370 \\
\hline 5.50 & 30.0 & 495 & 20.1 & .37 & ND & 515 & 1,460 \\
\hline 5.50 & 50.0 & 633 & 20.9 & .37 & ND & 654 & 1,620 \\
\hline 5.50 & 50.0 & 637 & 21.1 & .38 & ND & 658 & 1,660 \\
\hline 7.50 & 15.0 & $563^{*}$ & $22.9 *$ & $.44 *$ & ND* & $586^{*}$ & $920^{*}$ \\
\hline 7.50 & 15.0 & $578 *$ & $23.8 *$ & $.47 *$ & ND* & $602 *$ & $941^{*}$ \\
\hline 7.50 & 30.0 & 729 & 25.9 & .48 & ND & 755 & 1,210 \\
\hline 7.50 & 30.0 & 736 & 25.6 & .49 & ND & 762 & 1,240 \\
\hline 7.50 & 50.0 & 882 & 25.2 & .47 & ND & 908 & 1,240 \\
\hline 7.50 & 50.0 & 885 & 25.5 & .58 & ND & 911 & 1,270 \\
\hline 10.00 & 15.0 & 873 & 23.1 & .57 & ND & 897 & 619 \\
\hline 10.00 & 15.0 & 832 & 22.5 & .57 & ND & 855 & 642 \\
\hline 10.00 & 30.0 & 1,090 & 25.3 & .59 & ND & 1,120 & 728 \\
\hline 10.00 & 30.0 & 1,100 & 25.2 & .59 & ND & 1,130 & 804 \\
\hline 10.00 & 50.0 & 1,170 & 25.5 & .55 & ND & 1,200 & 795 \\
\hline 10.00 & 50.0 & 1,190 & 26.3 & .58 & ND & 1,220 & 865 \\
\hline
\end{tabular}

${ }^{*}$ Free-chlorine concentration less than the detection limit at reaction time of 168.0 hours. 
Table 5.--Concentrations of the four trihalomethane compounds and the total trihalomethane and nonpurgeable total organic-halide concentrations for a reaction time of 168.0 hours as a function of initial pH and initial free-chlorine concentration, Mississippi River at Davenport, lowa, October 2, 1991

$[\mu \mathrm{g} / \mathrm{L}$, microgram per liter; $\mathrm{CL}$, free-chlorine concentration; $\mathrm{mg} / \mathrm{L}$, milligram per liter; $\mathrm{CHCl}_{3}$, chloroform; $\mathrm{BrCHCl}_{2}$, bromodichloromethane; $\mathrm{ClCHBr}_{2}$, chlorodibromomethane; $\mathrm{CHBr}_{3}$, bromoform; $\mathrm{THM}$, trihalomethane; NPTOX, nonpurgeable total organic halide; ND, not detected]

\begin{tabular}{|c|c|c|c|c|c|c|c|}
\hline \multirow{2}{*}{ Initial pH } & \multirow{2}{*}{$\begin{array}{l}\text { Initial CL } \\
\text { (mg/L) }\end{array}$} & \multicolumn{6}{|c|}{$\begin{array}{c}\text { Concentration } \\
(\mu g / L)\end{array}$} \\
\hline & & $\mathrm{CHCl}_{3}$ & $\mathrm{BrCHCl}_{2}$ & $\mathrm{ClCHBr}_{2}$ & $\mathrm{CHBr}_{3}$ & $\begin{array}{l}\text { Total } \\
\text { THM }\end{array}$ & NPTOX \\
\hline 5.50 & 15.0 & 381 & 21.1 & 0.44 & $\mathrm{ND}$ & 403 & 1,180 \\
\hline 5.50 & 15.0 & 386 & 21.1 & .43 & ND & 408 & 1,160 \\
\hline 5.50 & 30.0 & 491 & 21.3 & .45 & ND & 513 & 1,460 \\
\hline 5.50 & 30.0 & 491 & 21.8 & .44 & ND & 513 & 1,480 \\
\hline 5.50 & 50.0 & 583 & 21.8 & .42 & ND & 605 & 1,540 \\
\hline 5.50 & 50.0 & 601 & 22.5 & .74 & ND & 624 & 1,540 \\
\hline 7.50 & 15.0 & 568 & 26.1 & .62 & ND & 595 & 917 \\
\hline 7.50 & 15.0 & 561 & 25.9 & .60 & ND & 588 & 875 \\
\hline 7.50 & 30.0 & 720 & 29.1 & .67 & ND & 750 & 1,150 \\
\hline 7.50 & 30.0 & 709 & 28.9 & .70 & ND & 739 & 1,200 \\
\hline 7.50 & 50.0 & 842 & 27.3 & .61 & ND & 870 & 1,190 \\
\hline 7.50 & 50.0 & 844 & 27.2 & .60 & ND & 872 & 1,210 \\
\hline 10.00 & 15.0 & 835 & 24.4 & .72 & ND & 860 & 606 \\
\hline 10.00 & 15.0 & 845 & 24.6 & .72 & ND & 870 & 644 \\
\hline 10.00 & 30.0 & 1,080 & 27.2 & .73 & ND & 1,110 & 754 \\
\hline 10.00 & 30.0 & 1,070 & 26.8 & .70 & ND & 1,100 & 752 \\
\hline 10.00 & 50.0 & 1,170 & 27.4 & .68 & ND & 1,200 & 815 \\
\hline 10.00 & 50.0 & 1,180 & 27.4 & .69 & ND & 1,210 & 834 \\
\hline
\end{tabular}


Table 6.-Concentrations of the four trihalomethane compounds and the total trihalomethane and nonpurgeable total organic-halide concentrations for a reaction time

of 168.0 hours as a function of initial pH and initial free-chlorine concentration, Mississippi River at Quincy, III., October 1, 1991

$[\mu \mathrm{g} / \mathrm{L}$, microgram per liter; $\mathrm{CL}$, free-chlorine concentration; $\mathrm{mg} / \mathrm{L}$, milligram per liter; $\mathrm{CHCl}_{3}$, chloroform; $\mathrm{BrCHCl}_{2}$, bromodichloromethane; $\mathrm{ClCHBr}_{2}$, chlorodibromomethane; $\mathrm{CHBr}_{3}$, bromoform; $\mathrm{THM}$, trihalomethane; NPTOX, nonpurgeable total organic halide; ND, not detected]

\begin{tabular}{|c|c|c|c|c|c|c|c|}
\hline \multirow{2}{*}{ Initial pH } & \multirow{2}{*}{$\begin{array}{l}\text { Initial CL } \\
\text { (mg/L) }\end{array}$} & \multicolumn{6}{|c|}{$\begin{array}{c}\text { Concentration } \\
(\mu g / L)\end{array}$} \\
\hline & & $\mathrm{CHCl}_{3}$ & $\mathrm{BrCHCl}_{2}$ & $\mathrm{ClCHBr}_{2}$ & $\mathrm{CHBr}_{3}$ & $\begin{array}{l}\text { Total } \\
\text { THM }\end{array}$ & NPTOX \\
\hline 5.50 & 15.0 & 337 & 23.2 & 0.67 & ND & 361 & 1,050 \\
\hline 5.50 & 15.0 & 336 & 22.1 & .66 & ND & 359 & 1,060 \\
\hline 5.50 & 30.0 & 440 & 24.1 & .69 & ND & 465 & 1,340 \\
\hline 5.50 & 30.0 & 439 & 23.9 & .65 & ND & 464 & 1,350 \\
\hline 5.50 & 50.0 & 554 & 25.8 & .71 & ND & 581 & 1,410 \\
\hline 5.50 & 50.0 & 554 & 25.5 & .65 & ND & 580 & 1,470 \\
\hline 7.50 & 15.0 & 539 & 30.3 & 1.04 & ND & 570 & 916 \\
\hline 7.50 & 15.0 & 553 & 30.7 & 1.05 & ND & 585 & 941 \\
\hline 7.50 & 30.0 & 664 & 34.0 & 1.07 & ND & 699 & 1,070 \\
\hline 7.50 & 30.0 & 662 & 34.2 & 1.03 & ND & 697 & 1,120 \\
\hline 7.50 & 50.0 & 812 & 32.9 & 1.08 & ND & 846 & 1,130 \\
\hline 7.50 & 50.0 & 809 & 32.3 & 1.01 & ND & 842 & 1,140 \\
\hline 10.00 & 15.0 & 807 & 30.7 & 1.34 & ND & 839 & 603 \\
\hline 10.00 & 15.0 & 800 & 30.0 & 1.22 & ND & 831 & 626 \\
\hline 10.00 & 30.0 & 949 & 32.7 & 1.16 & ND & 983 & 661 \\
\hline 10.00 & 30.0 & 958 & 32.6 & 1.14 & ND & 992 & 679 \\
\hline 10.00 & 50.0 & 797 & 30.0 & 1.21 & ND & 828 & 592 \\
\hline 10.00 & 50.0 & 778 & 29.1 & 1.18 & ND & 808 & 635 \\
\hline
\end{tabular}


Table 7.-Concentrations of the four trihalomethane compounds and the total trihalomethane and nonpurgeable total organlc-hallde concentrations for a reaction time of 168.0 hours as a function of initial pH and Initial free-chlorlne concentration, Missouri River 1.6 kilometers upstream from confluence with Mississippi River, September 30, 1991

$[\mu \mathrm{g} / \mathrm{L}$, microgram per liter; $\mathrm{CL}$, free-chlorine concentration; $\mathrm{mg} / \mathrm{L}$, milligram per liter; $\mathrm{CHCl}_{3}$, chloroform; $\mathrm{BrCHCl}_{2}$, bromodichloromethane; $\mathrm{ClCHBr}_{2}$, chlorodibromomethane; $\mathrm{CHBr}_{3}$, bromoform; $\mathrm{THM}$, trihalomethane; NPTOX, nonpurgeable total organic halide]

\begin{tabular}{|c|c|c|c|c|c|c|c|}
\hline \multirow{2}{*}{ Initial pH } & \multirow{2}{*}{$\begin{array}{c}\text { InitialCL } \\
\text { (mg/L) }\end{array}$} & \multicolumn{6}{|c|}{$\begin{array}{c}\text { Concentration } \\
(\mu \mathrm{g} / \mathrm{L})\end{array}$} \\
\hline & & $\mathrm{CHCl}_{3}$ & $\mathrm{BrCHCl}_{2}$ & $\mathrm{ClCHBr}_{2}$ & $\mathrm{CHBr}_{3}$ & $\begin{array}{l}\text { Total } \\
\text { THM }\end{array}$ & NPTOX \\
\hline 5.50 & 15.0 & 89.9 & 36.0 & 10.3 & 0.68 & 137 & 357 \\
\hline 5.50 & 15.0 & 90.6 & 35.7 & 10.1 & .67 & 137 & 372 \\
\hline 5.50 & 30.0 & 115 & 36.4 & 8.60 & .43 & 160 & 401 \\
\hline 5.50 & 30.0 & 115 & 34.8 & 8.21 & .44 & 158 & 411 \\
\hline 5.50 & 50.0 & 140 & 35.2 & 7.22 & .27 & 183 & 445 \\
\hline 5.50 & 50.0 & 145 & 36.5 & 7.51 & .29 & 189 & 448 \\
\hline 7.50 & 15.0 & 158 & 52.0 & 18.5 & 1.62 & 230 & 281 \\
\hline 7.50 & 15.0 & 157 & 53.0 & 19.2 & 1.65 & 231 & 284 \\
\hline 7.50 & 30.0 & 184 & 57.7 & 18.8 & 1.27 & 262 & 309 \\
\hline 7.50 & 30.0 & 182 & 57.5 & 18.9 & 1.29 & 260 & 351 \\
\hline 7.50 & 50.0 & 232 & 56.6 & 16.8 & 1.23 & 307 & 334 \\
\hline 7.50 & 50.0 & 253 & 59.8 & 16.9 & 1.31 & 331 & 367 \\
\hline 10.00 & 15.0 & 218 & 53.9 & 22.2 & 3.57 & 298 & 190 \\
\hline 10.00 & 15.0 & 213 & 52.7 & 21.5 & 3.49 & 291 & 223 \\
\hline 10.00 & 30.0 & 245 & 54.9 & 21.2 & 3.01 & 324 & 219 \\
\hline 10.00 & 30.0 & 253 & 55.6 & 21.1 & 2.99 & 333 & 232 \\
\hline 10.00 & 50.0 & 277 & 55.1 & 19.8 & 2.54 & 354 & 237 \\
\hline 10.00 & 50.0 & 284 & 56.4 & 20.8 & 2.55 & 364 & 256 \\
\hline
\end{tabular}


Table 8.-Concentrations of the four trihalomethane compounds and the total trihalomethane and nonpurgeable total organic-halide concentrations for a reaction time of 168.0 hours as a function of initial pH and initial free-chlorine concentration, Mississippi River at St. Louis, Mo., September 30, 1991

$[\mu \mathrm{g} / \mathrm{L}$, microgram per liter; $\mathrm{CL}$, free-chlorine concentration; $\mathrm{mg} / \mathrm{L}$, milligram per liter; $\mathrm{CHCl}_{3}$, chloroform; $\mathrm{BrCHCl}_{2}$, bromodichloromethane; $\mathrm{ClCHBr}_{2}$, chlorodibromomethane; $\mathrm{CHBr}_{3}$, bromoform; THM, trihalomethane; NPTOX, nonpurgeable total organic halide]

\begin{tabular}{|c|c|c|c|c|c|c|c|}
\hline \multirow{2}{*}{ Initial pH } & \multirow{2}{*}{$\begin{array}{l}\text { Initial CL } \\
\text { (mg/L) }\end{array}$} & \multicolumn{6}{|c|}{$\begin{array}{c}\text { Concentration } \\
(\mu \mathrm{g} / \mathrm{L})\end{array}$} \\
\hline & & $\mathrm{CHCl}_{3}$ & $\mathrm{BrCHCl}_{2}$ & $\mathrm{ClCHBr}_{2}$ & $\mathrm{CHBr}_{3}$ & $\begin{array}{l}\text { Total } \\
\text { THM }\end{array}$ & NPTOX \\
\hline 5.50 & 15.0 & 181 & 35.2 & 5.55 & 0.15 & 222 & 596 \\
\hline 5.50 & 15.0 & 182 & 35.5 & 5.59 & .16 & 223 & 626 \\
\hline 5.50 & 30.0 & 220 & 35.8 & 4.74 & .10 & 261 & 690 \\
\hline 5.50 & 30.0 & 225 & 37.2 & 4.81 & .10 & 267 & 685 \\
\hline 5.50 & 50.0 & 270 & 37.7 & 4.46 & .06 & 312 & 787 \\
\hline 5.50 & 50.0 & 269 & 36.5 & 4.38 & .07 & 310 & 775 \\
\hline 7.50 & 15.0 & 290 & 53.7 & 8.29 & .24 & 352 & 482 \\
\hline 7.50 & 15.0 & 294 & 52.3 & 8.23 & .25 & 355 & 485 \\
\hline 7.50 & 30.0 & 350 & 64.5 & 9.07 & .21 & 424 & 549 \\
\hline 7.50 & 30.0 & 344 & 60.8 & 8.55 & .19 & 414 & 551 \\
\hline 7.50 & 50.0 & 415 & 59.1 & 8.19 & .19 & 482 & 546 \\
\hline 7.50 & 50.0 & 409 & 58.0 & 8.11 & .19 & 475 & 551 \\
\hline 10.00 & 15.0 & 398 & 53.6 & 9.60 & .52 & 462 & 306 \\
\hline 10.00 & 15.0 & 400 & 53.9 & 9.63 & .53 & 464 & 314 \\
\hline 10.00 & 30.0 & 442 & 54.9 & 9.11 & .42 & 506 & 350 \\
\hline 10.00 & 30.0 & 443 & 55.5 & 9.34 & .43 & 508 & 391 \\
\hline 10.00 & 50.0 & 466 & 55.1 & 8.92 & .38 & 530 & 372 \\
\hline 10.00 & 50.0 & 479 & 57.7 & 9.26 & .40 & 546 & 373 \\
\hline
\end{tabular}


Table 9.-Concentrations of the four trihalomethane compounds and the total trihalomethane and nonpurgeable total organic-halide concentrations for a reaction time of 168.0 hours as a function of initial pH and initial free-chlorine concentration, Mississippi River at Cairo, III., September 30, 1991

$[\mu \mathrm{g} / \mathrm{L}$, microgram per liter; $\mathrm{CL}$, free-chlorine concentration; $\mathrm{mg} / \mathrm{L}$, milligram per liter; $\mathrm{CHCl}_{3}$, chloroform; $\mathrm{BrCHCl}_{2}$, bromodichloromethane; $\mathrm{ClCHBr}_{2}$, chlorodibromomethane; $\mathrm{CHBr}_{3}$, bromoform; $\mathrm{THM}$, trihalomethane; NPTOX, nonpurgeable total organic halide; ND, not detected]

\begin{tabular}{|c|c|c|c|c|c|c|c|}
\hline \multirow{2}{*}{ Initial pH } & \multirow{2}{*}{$\begin{array}{c}\text { Initial CL } \\
\text { (mg/L) }\end{array}$} & \multicolumn{6}{|c|}{$\begin{array}{c}\text { Concentration } \\
(\mu \mathrm{g} / \mathrm{L})\end{array}$} \\
\hline & & $\mathrm{CHCl}_{3}$ & $\mathrm{BrCHCl}_{2}$ & $\mathrm{ClCHBr}_{2}$ & $\mathrm{CHBr}_{3}$ & $\begin{array}{l}\text { Total } \\
\text { THM }\end{array}$ & NPTOX \\
\hline 5.50 & 15.0 & 258 & 35.0 & 3.30 & 0.11 & 296 & 789 \\
\hline 5.50 & 15.0 & 256 & 34.3 & 3.23 & .10 & 294 & 786 \\
\hline 5.50 & 30.0 & 315 & 36.3 & 3.46 & .04 & 355 & 886 \\
\hline 5.50 & 30.0 & 357 & 39.7 & 3.86 & ND & 401 & 843 \\
\hline 5.50 & 50.0 & 369 & 36.4 & 3.13 & ND & 409 & 705 \\
\hline 5.50 & 50.0 & 364 & 35.2 & 2.96 & ND & 402 & 785 \\
\hline 7.50 & 15.0 & 366 & 47.5 & 5.54 & .10 & 419 & 542 \\
\hline 7.50 & 15.0 & 380 & 48.5 & 5.65 & .10 & 434 & 606 \\
\hline 7.50 & 30.0 & 449 & 54.9 & 5.91 & .08 & 510 & 730 \\
\hline 7.50 & 30.0 & 437 & 54.2 & 5.85 & .08 & 497 & 723 \\
\hline 7.50 & 50.0 & 540 & 51.0 & 5.35 & .08 & 596 & 716 \\
\hline 7.50 & 50.0 & 544 & 52.2 & 5.46 & .08 & 602 & 698 \\
\hline 10.00 & 15.0 & 530 & 48.4 & 6.63 & .24 & 585 & 411 \\
\hline 10.00 & 15.0 & 535 & 49.8 & 6.90 & .26 & 592 & 419 \\
\hline 10.00 & 30.0 & 633 & 55.2 & 7.05 & .22 & 695 & 442 \\
\hline 10.00 & 30.0 & 602 & 49.6 & 6.19 & .19 & 658 & 456 \\
\hline 10.00 & 50.0 & 658 & 52.5 & 6.45 & .19 & 717 & 464 \\
\hline 10.00 & 50.0 & 656 & 52.1 & 6.35 & .18 & 715 & 499 \\
\hline
\end{tabular}


Table 10.-Concentrations of the four trihalomethane compounds and the total trihalomethane and nonpurgeable total organic-halide concentrations for a reaction time of 168.0 hours as a function of initial pH and initial free-chlorine concentration, Ohlo River 1.6 kilometers upstream from confluence with Mississippi River, September 29, 1991

$[\mu \mathrm{g} / \mathrm{L}$, microgram per liter; $\mathrm{CL}$, free-chlorine concentration; $\mathrm{mg} / \mathrm{L}$, milligram per liter; $\mathrm{CHCl}_{3}$, chloroform; $\mathrm{BrCHCl}_{2}$, bromodichloromethane; $\mathrm{ClCHBr}_{2}$, chlorodibromomethane; $\mathrm{CHBr}_{3}$, bromoform; $\mathrm{THM}$, trihalomethane; NPTOX, nonpurgeable total organic halide]

\begin{tabular}{|c|c|c|c|c|c|c|c|}
\hline \multirow{2}{*}{ Initial pH } & \multirow{2}{*}{$\begin{array}{c}\text { Initial CL } \\
\text { (mg/l) }\end{array}$} & \multicolumn{6}{|c|}{$\begin{array}{c}\text { Concentration } \\
(\mu g / L)\end{array}$} \\
\hline & & $\mathrm{CHCl}_{3}$ & $\mathrm{BrCHCl}_{2}$ & $\mathrm{ClCHBr}_{2}$ & $\mathrm{CHBr}_{3}$ & $\begin{array}{l}\text { Total } \\
\text { THM }\end{array}$ & NPTOX \\
\hline 5.50 & 15.0 & 92.8 & 26.6 & 5.44 & 0.21 & 125 & 338 \\
\hline 5.50 & 15.0 & 92.3 & 26.5 & 5.41 & .22 & 124 & 350 \\
\hline 5.50 & 30.0 & 126 & 27.3 & 4.77 & .15 & 158 & 367 \\
\hline 5.50 & 30.0 & 130 & 28.3 & 4.87 & .15 & 163 & 355 \\
\hline 5.50 & 50.0 & 166 & 29.4 & 4.76 & .13 & 200 & 376 \\
\hline 5.50 & 50.0 & 167 & 30.0 & 4.88 & .13 & 202 & 405 \\
\hline 7.50 & 15.0 & 143 & 34.0 & 8.88 & .57 & 186 & 250 \\
\hline 7.50 & 15.0 & 161 & 37.5 & 9.38 & .61 & 208 & 258 \\
\hline 7.50 & 30.0 & 202 & 39.8 & 9.31 & .53 & 252 & 291 \\
\hline 7.50 & 30.0 & 197 & 39.8 & 9.61 & .55 & 247 & 270 \\
\hline 7.50 & 50.0 & 237 & 39.5 & 8.96 & .51 & 286 & 276 \\
\hline 7.50 & 50.0 & 237 & 40.7 & 9.45 & .54 & 288 & 280 \\
\hline 10.00 & 15.0 & 216 & 36.2 & 10.5 & 1.12 & 264 & 169 \\
\hline 10.00 & 15.0 & 215 & 36.4 & 10.4 & 1.11 & 263 & 181 \\
\hline 10.00 & 30.0 & 242 & 38.1 & 10.5 & .97 & 292 & 163 \\
\hline 10.00 & 30.0 & 240 & 37.3 & 10.2 & .93 & 288 & 180 \\
\hline 10.00 & 50.0 & 272 & 40.1 & 10.5 & .86 & 323 & 186 \\
\hline 10.00 & 50.0 & 271 & 38.7 & 10.0 & .81 & 321 & 199 \\
\hline
\end{tabular}


Table 11.-Concentrations of the four trihalomethane compounds and the total trihalomethane and nonpurgeable total organic-halide concentrations for a reaction time of $\mathbf{1 6 8 . 0}$ hours as a function of initial $\mathrm{pH}$ and initial free-chlorine concentration, Mississippi River at Memphis, Tenn., September 28, 1991

$[\mu \mathrm{g} / \mathrm{L}$, microgram per liter; $\mathrm{CL}$, free-chlorine concentration; $\mathrm{mg} / \mathrm{L}$, milligram per liter; $\mathrm{CHCl}_{3}$, chloroform; $\mathrm{BrCHCl}_{2}$, bromodichloromethane; $\mathrm{ClCHBr}_{2}$, chlorodibromomethane; $\mathrm{CHBr}_{3}$, bromoform; $\mathrm{THM}$, trihalomethane; NPTOX, nonpurgeable total organic halide]

\begin{tabular}{|c|c|c|c|c|c|c|c|}
\hline \multirow{2}{*}{ Initial pH } & \multirow{2}{*}{$\begin{array}{c}\text { Initial CL } \\
\text { (mg/l) }\end{array}$} & \multicolumn{6}{|c|}{$\begin{array}{c}\text { Concentration } \\
(\mu g / L)\end{array}$} \\
\hline & & $\mathrm{CHCl}_{3}$ & $\mathrm{BrCHCl}_{2}$ & $\mathrm{ClCHBr}_{2}$ & $\mathrm{CHBr}_{3}$ & $\begin{array}{l}\text { Total } \\
\text { THM }\end{array}$ & NPTOX \\
\hline 5.50 & 15.0 & 172 & 34.0 & 4.45 & 0.12 & 211 & 559 \\
\hline 5.50 & 15.0 & 165 & 32.1 & 4.19 & .11 & 201 & 568 \\
\hline 5.50 & 30.0 & 217 & 34.5 & 3.80 & .09 & 255 & 659 \\
\hline 5.50 & 30.0 & 216 & 33.9 & 3.69 & .07 & 254 & 67.5 \\
\hline 5.50 & 50.0 & 280 & 37.4 & 3.81 & .06 & 321 & 791 \\
\hline 5.50 & 50.0 & 272 & 35.5 & 3.68 & .05 & 311 & 752 \\
\hline 7.50 & 15.0 & 277 & 48.0 & 7.38 & .18 & 333 & 444 \\
\hline 7.50 & 15.0 & 271 & 46.2 & 6.99 & .18 & 324 & 468 \\
\hline 7.50 & 30.0 & 322 & 51.8 & 7.40 & .15 & 381 & 515 \\
\hline 7.50 & 30.0 & 333 & 52.8 & 7.52 & .15 & 393 & 541 \\
\hline 7.50 & 50.0 & 388 & 50.0 & 7.00 & .13 & 445 & 536 \\
\hline 7.50 & 50.0 & 388 & 49.9 & 6.82 & .14 & 445 & 544 \\
\hline 10.00 & 15.0 & 375 & 44.0 & 7.65 & .38 & 427 & 315 \\
\hline 10.00 & 15.0 & 373 & 44.1 & 7.65 & .38 & 425 & 305 \\
\hline 10.00 & 30.0 & 424 & 45.5 & 7.27 & .30 & 477 & 343 \\
\hline 10.00 & 30.0 & 433 & 48.0 & 7.75 & .32 & 489 & 340 \\
\hline 10.00 & 50.0 & 463 & 48.4 & 7.52 & .28 & 519 & 404 \\
\hline 10.00 & 50.0 & 465 & 48.7 & 7.52 & .28 & 522 & 403 \\
\hline
\end{tabular}


Table 12.-Concentrations of the four trihalomethane compounds and the total trihalomethane and nonpurgeable total organic-halide concentrations for a reaction time of 168.0 hours as a function of initial pH and initial free-chlorine concentration, Mississippi River at Greenville, Miss., September 27, 1991

$[\mu \mathrm{g} / \mathrm{L}$, microgram per liter; $\mathrm{CL}$, free-chlorine concentration; $\mathrm{mg} / \mathrm{L}$, milligram per liter; $\mathrm{CHCl}_{3}$, chloroform; $\mathrm{BrCHCl}_{2}$, bromodichloromethane; $\mathrm{ClCHBr}$, chlorodibromomethane; $\mathrm{CHBr}_{3}$, bromoform; $\mathrm{THM}$, trihalomethane; NPTOX, nonpurgeable total organic halide]

\begin{tabular}{|c|c|c|c|c|c|c|c|}
\hline \multirow{2}{*}{ Initial pH } & \multirow{2}{*}{$\begin{array}{l}\text { Initial CL } \\
\text { (mg/L) }\end{array}$} & \multicolumn{6}{|c|}{$\begin{array}{c}\text { Concentration } \\
(\mu g / L)\end{array}$} \\
\hline & & $\mathrm{CHCl}_{3}$ & $\mathrm{BrCHCl}_{2}$ & $\mathrm{CICHBr}_{2}$ & $\mathrm{CHBr}_{3}$ & $\begin{array}{l}\text { Total } \\
\text { THM }\end{array}$ & NPTOX \\
\hline 5.50 & 15.0 & 153 & 33.2 & 4.94 & 0.17 & 191 & 468 \\
\hline 5.50 & 15.0 & 149 & 32.5 & 5.01 & .16 & 187 & 572 \\
\hline 5.50 & 30.0 & 197 & 35.2 & 4.48 & .15 & 237 & 571 \\
\hline 5.50 & 30.0 & 197 & 35.3 & 4.67 & .17 & 237 & 688 \\
\hline 5.50 & 50.0 & 246 & 36.5 & 4.35 & .13 & 287 & 669 \\
\hline 5.50 & 50.0 & 241 & 35.1 & 4.21 & .12 & 280 & 715 \\
\hline 7.50 & 15.0 & 248 & 47.6 & 8.22 & .30 & 304 & 409 \\
\hline 7.50 & 15.0 & 245 & 45.2 & 7.76 & .28 & 298 & 390 \\
\hline 7.50 & 30.0 & 311 & 56.5 & 9.25 & .22 & 377 & 433 \\
\hline 7.50 & 30.0 & 307 & 56.4 & 9.20 & .22 & 373 & 463 \\
\hline 7.50 & 50.0 & 366 & 50.4 & 7.69 & .20 & 424 & 480 \\
\hline 7.50 & 50.0 & 374 & 54.8 & 8.10 & .27 & 437 & 454 \\
\hline 10.00 & 15.0 & 358 & 47.4 & 9.02 & .58 & 415 & 243 \\
\hline 10.00 & 15.0 & 364 & 47.7 & 9.04 & .57 & 421 & 243 \\
\hline 10.00 & 30.0 & 407 & 47.7 & 8.41 & .46 & 464 & 282 \\
\hline 10.00 & 30.0 & 426 & 50.1 & 8.79 & .48 & 485 & 317 \\
\hline 10.00 & 50.0 & 465 & 52.3 & 8.93 & .60 & 527 & 306 \\
\hline 10.00 & 50.0 & 458 & 50.0 & 8.62 & .47 & 517 & 316 \\
\hline
\end{tabular}


Table 13.-Concentrations of the four trihalomethane compounds and the total trihalomethane and nonpurgeable total organic-halide concentrations for a reaction time of 168.0 hours as a function of initial pH and initial free-chlorine concentration, Mississippi River at Natchez, Miss., September 26, 1991

$[\mu \mathrm{g} / \mathrm{L}$, microgram per liter; $\mathrm{CL}$, free-chlorine concentration; $\mathrm{mg} / \mathrm{L}$, milligram per liter; $\mathrm{CHCl}_{3}$, chloroform; $\mathrm{BrCHCl}_{2}$, bromodichloromethane; $\mathrm{ClCHBr}_{2}$, chlorodibromomethane; $\mathrm{CHBr}_{3}$, bromoform; THM, trihalomethane; NPTOX, nonpurgeable total organic halide]

\begin{tabular}{|c|c|c|c|c|c|c|c|}
\hline \multirow{2}{*}{ Initial pH } & \multirow{2}{*}{$\begin{array}{l}\text { Initial CL } \\
\text { (mg/L) }\end{array}$} & \multicolumn{6}{|c|}{$\begin{array}{c}\text { Concentration } \\
(\mu g / L)\end{array}$} \\
\hline & & $\mathrm{CHCl}_{3}$ & $\mathrm{BrCHCl}_{2}$ & $\mathrm{ClCHBr}_{2}$ & $\mathrm{CHBr}_{3}$ & $\begin{array}{l}\text { Total } \\
\text { THM }\end{array}$ & NPTOX \\
\hline 5.50 & 15.0 & 146 & 32.8 & 5.22 & 0.16 & 184 & 412 \\
\hline 5.50 & 15.0 & 140 & 31.3 & 4.99 & .15 & 176 & 436 \\
\hline 5.50 & 30.0 & 176 & 32.8 & 4.51 & .09 & 213 & 531 \\
\hline 5.50 & 30.0 & 177 & 33.6 & 4.66 & .09 & 215 & 472 \\
\hline 5.50 & 50.0 & 215 & 34.0 & 4.32 & .07 & 253 & 495 \\
\hline 5.50 & 50.0 & 216 & 34.1 & 4.26 & .06 & 254 & 507 \\
\hline 7.50 & 15.0 & 237 & 51.4 & 9.80 & .37 & 299 & 342 \\
\hline 7.50 & 15.0 & 232 & 48.0 & 8.86 & .34 & 289 & 343 \\
\hline 7.50 & 30.0 & 278 & 50.7 & 8.91 & .29 & 338 & 406 \\
\hline 7.50 & 30.0 & 276 & 49.8 & 8.72 & .28 & 335 & 389 \\
\hline 7.50 & 50.0 & 328 & 52.5 & 8.67 & .28 & 389 & 370 \\
\hline 7.50 & 50.0 & 328 & 52.0 & 8.56 & .28 & 389 & 429 \\
\hline 10.00 & 15.0 & 311 & 45.0 & 9.32 & .64 & 366 & 260 \\
\hline 10.00 & 15.0 & 304 & 43.8 & 8.92 & .60 & 357 & 247 \\
\hline 10.00 & 30.0 & 343 & 46.2 & 8.81 & .50 & 399 & 250 \\
\hline 10.00 & 30.0 & 351 & 47.5 & 9.05 & .52 & 408 & 237 \\
\hline 10.00 & 50.0 & 373 & 47.1 & 8.73 & .45 & 429 & 286 \\
\hline 10.00 & 50.0 & 378 & 46.5 & 8.52 & .43 & 433 & 304 \\
\hline
\end{tabular}


Table 14.-Concentrations of the four trihalomethane compounds and the total trihalomethane and nonpurgeable total organic-halide concentrations for a reaction time of 168.0 hours as a function of Initial pH and InItlal free-chlorine concentration, Mississippl River at Baton Rouge, La., September 26, 1991

$[\mu \mathrm{g} / \mathrm{L}$, microgram per liter; $\mathrm{CL}$, free-chlorine concentration; $\mathrm{mg} / \mathrm{L}$, milligram per liter; $\mathrm{CHCl}_{3}$, chloroform; $\mathrm{BrCHCl}_{2}$, bromodichloromethane; $\mathrm{ClCHBr}_{2}$, chlorodibromomethane; $\mathrm{CHBr}_{3}$, bromoform; $\mathrm{THM}$, trihalomethane; NPTOX, nonpurgeable total organic halide]

\begin{tabular}{|c|c|c|c|c|c|c|c|}
\hline \multirow{2}{*}{ Initial pH } & \multirow{2}{*}{$\begin{array}{c}\text { Initial CL } \\
\text { (mg/L) }\end{array}$} & \multicolumn{6}{|c|}{$\begin{array}{c}\text { Concentration } \\
(\mu g / L)\end{array}$} \\
\hline & & $\mathrm{CHCl}_{3}$ & $\mathrm{BrCHCl}_{2}$ & $\mathrm{ClCHBr}_{2}$ & $\mathrm{CHBr}_{3}$ & $\begin{array}{l}\text { Total } \\
\text { THM }\end{array}$ & NPTOX \\
\hline 5.50 & 15.0 & 140 & 36.6 & 7.18 & 0.27 & 184 & 480 \\
\hline 5.50 & 15.0 & 136 & 35.1 & 6.90 & .27 & 178 & 408 \\
\hline 5.50 & 30.0 & 169 & 35.1 & 6.18 & .16 & 210 & 512 \\
\hline 5.50 & 30.0 & 170 & 36.1 & 6.33 & .18 & 213 & 535 \\
\hline 5.50 & 50.0 & 216 & 39.8 & 6.35 & .15 & 262 & 541 \\
\hline 5.50 & 50.0 & 211 & 38.2 & 6.15 & .15 & 256 & 542 \\
\hline 7.50 & 15.0 & 209 & 45.0 & 9.91 & .46 & 264 & 337 \\
\hline 7.50 & 15.0 & 217 & 45.8 & 10.0 & .46 & 273 & 360 \\
\hline 7.50 & 30.0 & 230 & 46.8 & 9.96 & .41 & 287 & 398 \\
\hline 7.50 & 30.0 & 239 & 47.4 & 10.1 & .41 & 297 & 369 \\
\hline 7.50 & 50.0 & 305 & 49.3 & 9.75 & .39 & 364 & 418 \\
\hline 7.50 & 50.0 & 308 & 52.4 & 10.6 & .42 & 371 & 394 \\
\hline 10.00 & 15.0 & 293 & 49.6 & 12.4 & .95 & 356 & 216 \\
\hline 10.00 & 15.0 & 292 & 47.8 & 11.9 & .86 & 353 & 238 \\
\hline 10.00 & 30.0 & 348 & 53.8 & 12.6 & .81 & 415 & 295 \\
\hline 10.00 & 30.0 & 339 & 52.4 & 12.2 & .79 & 404 & 255 \\
\hline 10.00 & 50.0 & 369 & 53.3 & 12.0 & .74 & 435 & 268 \\
\hline 10.00 & 50.0 & 386 & 53.8 & 12.1 & .70 & 453 & 258 \\
\hline
\end{tabular}


Table 15.-Concentrations of the four trihalomethane compounds and the total trihalomethane and nonpurgeable total organic-halide concentrations for a reaction time of 168.0 hours as a function of initial pH and initial free-chlorine concentration, Mississippi River at New Orleans, La., September 25, 1991

$[\mu \mathrm{g} / \mathrm{L}$, microgram per liter; $\mathrm{CL}$, free-chlorine concentration; $\mathrm{mg} / \mathrm{L}$, milligram per liter; $\mathrm{CHCl}_{3}$, chloroform; $\mathrm{BrCHCl}_{2}$, bromodichloromethane; $\mathrm{ClCHBr}_{2}$, chlorodibromomethane; $\mathrm{CHBr}_{3}$, bromoform; $\mathrm{THM}$, trihalomethane; NPTOX, nonpurgeable total organic halide]

\begin{tabular}{|c|c|c|c|c|c|c|c|}
\hline \multirow{2}{*}{ Initial pH } & \multirow{2}{*}{$\begin{array}{c}\text { Initial CL } \\
\text { (mg/L) }\end{array}$} & \multicolumn{6}{|c|}{$\begin{array}{c}\text { Concentration } \\
(\mu g / L)\end{array}$} \\
\hline & & $\mathrm{CHCl}_{3}$ & $\mathrm{BrCHCl}_{2}$ & $\mathrm{ClCHBr}_{2}$ & $\mathrm{CHBr}_{3}$ & $\begin{array}{l}\text { Total } \\
\text { THM }\end{array}$ & NPTOX \\
\hline 5.50 & 15.0 & 148 & 34.9 & 6.07 & 0.23 & 189 & 520 \\
\hline 5.50 & 15.0 & 151 & 38.1 & 6.73 & .26 & 196 & 523 \\
\hline 5.50 & 30.0 & 181 & 39.9 & 6.27 & .19 & 227 & 395 \\
\hline 5.50 & 30.0 & 179 & 38.2 & 5.87 & .18 & 223 & 418 \\
\hline 5.50 & 50.0 & 215 & 41.5 & 5.82 & .15 & 262 & 611 \\
\hline 5.50 & 50.0 & 216 & 41.9 & 5.70 & .14 & 264 & 596 \\
\hline 7.50 & 15.0 & 215 & 41.7 & 11.5 & .66 & 269 & 393 \\
\hline 7.50 & 15.0 & 220 & 42.7 & 11.4 & .64 & 275 & 390 \\
\hline 7.50 & 30.0 & 251 & 53.7 & 11.0 & .49 & 316 & 439 \\
\hline 7.50 & 30.0 & 255 & 54.9 & 11.4 & .50 & 322 & 445 \\
\hline 7.50 & 50.0 & 323 & 59.8 & 11.4 & .50 & 395 & 521 \\
\hline 7.50 & 50.0 & 311 & 54.5 & 10.1 & .42 & 376 & 434 \\
\hline 10.00 & 15.0 & 302 & 55.8 & 13.3 & 1.17 & 372 & 308 \\
\hline 10.00 & 15.0 & 306 & 55.4 & 13.1 & 1.14 & 376 & 275 \\
\hline 10.00 & 30.0 & 348 & 62.2 & 13.8 & 1.06 & 425 & 296 \\
\hline 10.00 & 30.0 & 347 & 60.6 & 13.4 & 1.02 & 422 & 287 \\
\hline 10.00 & 50.0 & 377 & 58.5 & 12.3 & .81 & 449 & 329 \\
\hline 10.00 & 50.0 & 386 & 61.7 & 13.0 & .88 & 462 & 321 \\
\hline
\end{tabular}


Table 16.-Concentrations of the four trihalomethane compounds and the total trihalomethane and nonpurgeable total organic-halide concentrations as a function of reaction time for an initial pH of 7.50 and an initial free-chlorlne concentration of 30.0 milligrams per liter, Mississippi River at Minneapolis, Minn., October 7, 1991

[ $\mu \mathrm{g} / \mathrm{L}$, microgram per liter; $\mathrm{CHCl}_{3}$, chloroform; $\mathrm{BrCHCl}_{2}$, bromodichloromethane; $\mathrm{ClCHBr}_{2}$, chlorodibromomethane; $\mathrm{CHBr}_{3}$, bromoform; $\mathrm{THM}$, trihalomethane;

NPTOX, nonpurgeable total organic halide; ND, not detected]

\begin{tabular}{ccccccc}
\hline $\begin{array}{c}\text { Reaction } \\
\text { time } \\
\text { (hours) }\end{array}$ & $\mathrm{CHCl}_{3}$ & $\mathrm{BrCHCl}_{2}$ & $\mathrm{ClCHBr}_{2}$ & $\mathrm{CHBr}_{3}$ & Total THM & NPTOX \\
\cline { 2 - 7 } & 77.5 & 5.33 & 0.07 & $\mathrm{ND}$ & 82.9 & 300 \\
\hline 0.00 & 283 & 19.9 & .27 & $\mathrm{ND}$ & 303 & 794 \\
2.00 & 369 & 23.0 & .33 & $\mathrm{ND}$ & 392 & 998 \\
6.22 & 422 & 24.3 & .35 & $\mathrm{ND}$ & 447 & 1,280 \\
12.00 & 516 & 27.0 & .41 & $\mathrm{ND}$ & 543 & 1,280 \\
22.78 & & & & & & \\
& & 28.9 & .56 & ND & 655 & 1,480 \\
47.00 & 626 & .45 & ND & 731 & 1,530 \\
72.00 & 701 & 29.6 & .45 & ND & 811 & 1,600 \\
125.0 & 781 & 29.1 & .47 & ND & 905 & 1,640 \\
168.0 & 873 & 31.1 & .47 & ND & 914 & 1,620 \\
168.0 & 883 & 31.0 & & &
\end{tabular}

Table 17.-Concentrations of the four trihalomethane compounds and the total trihalomethane and nonpurgeable total organic-halide concentrations as a function of reaction time for an initial pH of 7.50 and an initial free-chlorine concentration of 30.0 milligrams per liter, Mississippi River at LaCrosse, Wis., October 4, 1991 [ $\mu \mathrm{g} / \mathrm{L}$, microgram per liter; $\mathrm{CHCl}_{3}$, chloroform; $\mathrm{BrCHCl}_{2}$, bromodichloromethane; $\mathrm{ClCHBr}_{2}$, chlorodibromomethane; $\mathrm{CHBr}_{3}$, bromoform; $\mathrm{THM}$, trihalomethane; NPTOX, nonpurgeable total organic halide; ND, not detected]

\begin{tabular}{ccccccc}
\hline $\begin{array}{c}\text { Reaction } \\
\text { time } \\
\text { (hours) }\end{array}$ & $\mathrm{CHCl}_{3}$ & $\mathrm{BrCHCl}_{2}$ & $\mathrm{ClCHBr}_{2}$ & $\mathrm{CHBr}_{3}$ & Total THM & NPTOX \\
\cline { 2 - 7 } & 64.5 & 4.21 & 0.07 & $\mathrm{ND}$ & 68.8 & 225 \\
0.00 & 253 & 16.5 & .23 & $\mathrm{ND}$ & 270 & 709 \\
2.00 & 336 & 19.8 & .33 & $\mathrm{ND}$ & 356 & 842 \\
6.00 & 387 & 20.4 & .36 & $\mathrm{ND}$ & 408 & 1,020 \\
12.00 & 485 & 23.4 & .43 & $\mathrm{ND}$ & 509 & 1,150 \\
24.00 & & & & & & \\
& 592 & 22.3 & .41 & ND & 615 & 1,170 \\
48.00 & 666 & 26.4 & .49 & ND & 693 & 1,240 \\
72.00 & 758 & 27.5 & .51 & ND & 786 & 1,380 \\
121.0 & 822 & 28.2 & .52 & ND & 851 & 1,390 \\
168.0 & 818 & 27.8 & .51 & ND & 846 & 1,360 \\
168.0 & & & & & & \\
\hline
\end{tabular}


Table 18.-Concentrations of the four trihalomethane compounds and the total trihalomethane and nonpurgeable total organic-hallde concentrations as a function of reaction time for an initial pH of 7.50 and an Initial free-chlorine concentration of 30.0 milligrams per liter, Mississippi River at Dubuque, lowa, October 3, 1991

$\left[\mu \mathrm{g} / \mathrm{L}\right.$, microgram per liter; $\mathrm{CHCl}_{3}$, chloroform; $\mathrm{BrCHCl}_{2}$, bromodichloromethane; $\mathrm{ClCHBr}_{2}$, chlorodibromomethane; $\mathrm{CHBr}_{3}$, bromoform; $\mathrm{THM}$, trihalomethane; NPTOX, nonpurgeable total organic halide; ND, not detected]

\begin{tabular}{ccccccc}
\hline $\begin{array}{c}\text { Reaction } \\
\text { time } \\
\text { (hours) }\end{array}$ & $\mathrm{CHCl}_{3}$ & $\mathrm{BrCHCl}_{2}$ & $\mathrm{ClCHBr}_{2}$ & $\mathrm{CHBr}_{3}$ & Total THM & NPTOX \\
\cline { 2 - 7 } & 56.7 & 3.71 & 0.03 & $\mathrm{ND}$ & 60.4 & 211 \\
0.00 & 223 & 15.8 & .26 & $\mathrm{ND}$ & 239 & 591 \\
2.00 & 278 & 17.6 & .33 & $\mathrm{ND}$ & 296 & 747 \\
6.00 & 341 & 20.3 & .38 & $\mathrm{ND}$ & 362 & 907 \\
12.00 & 427 & 22.2 & .44 & $\mathrm{ND}$ & 450 & 975 \\
24.00 & & & & & & \\
& 510 & 23.1 & .44 & $\mathrm{ND}$ & 534 & 1,100 \\
48.00 & 585 & 24.8 & .48 & $\mathrm{ND}$ & 610 & 1,150 \\
72.00 & 661 & 24.2 & .45 & $\mathrm{ND}$ & 686 & 1,200 \\
125.0 & 729 & 25.9 & .48 & $\mathrm{ND}$ & 755 & 1,210 \\
168.0 & 736 & 25.6 & .49 & $\mathrm{ND}$ & 762 & 1,240 \\
168.0 & & & & & &
\end{tabular}

Table 19.-Concentrations of the four trihalomethane compounds and the total trihalomethane and nonpurgeable total organic-halide concentrations as a function of reaction time for an initial pH of 7.50 and an initial free-chlorine concentration of 30.0 milligrams per liter, Mississippi River at Davenport, lowa, October 2, 1991

[ $\mu \mathrm{g} / \mathrm{L}$, microgram per liter; $\mathrm{CHCl}_{3}$, chloroform; $\mathrm{BrCHCl}_{2}$, bromodichloromethane; $\mathrm{ClCHBr}_{2}$, chlorodibromomethane; $\mathrm{CHBr}_{3}$, bromoform; $\mathrm{THM}$, trihalomethane; NPTOX, nonpurgeable total organic halide; ND, not detected]

\begin{tabular}{ccccccc}
\hline $\begin{array}{c}\text { Reaction } \\
\text { time } \\
\text { (hours) }\end{array}$ & $\mathrm{CHCl}_{3}$ & $\mathrm{BrCHCl}_{2}$ & $\mathrm{ClCHBr}_{2}$ & $\mathrm{CHBr}_{3}$ & Total THM & NPTOX \\
\hline 0.00 & 56.6 & 4.60 & 0.08 & $\mathrm{ND}$ & 61.3 & 195 \\
2.00 & 221 & 17.9 & .35 & $\mathrm{ND}$ & 239 & 551 \\
6.00 & 278 & 20.9 & .43 & $\mathrm{ND}$ & 299 & 743 \\
13.05 & 345 & 23.0 & .51 & ND & 369 & 852 \\
24.00 & 417 & 25.0 & .60 & ND & 443 & 954 \\
& & & & & & \\
48.00 & 504 & 26.2 & .62 & ND & 531 & 1,030 \\
72.00 & 554 & 26.9 & .62 & ND & 582 & 1,080 \\
120.1 & 629 & 27.6 & .63 & ND & 657 & 1,110 \\
168.0 & 720 & 29.1 & .67 & ND & 750 & 1,150 \\
168.0 & 709 & 28.9 & .70 & ND & 739 & 1,200 \\
\hline
\end{tabular}


Table 20.-Concentrations of the four trihalomethane compounds and the total trihalomethane and nonpurgeable total organic-halide concentrations as a function of reaction time for an initial pH of 7.50 and an initial free-chlorine concentration of 30.0 milligrams per liter, Mississippi River at Quincy, III., October 1, 1991

[ $\mu \mathrm{g} / \mathrm{L}$, microgram per liter; $\mathrm{CHCl}_{3}$, chloroform; $\mathrm{BrCHCl}_{2}$, bromodichloromethane; $\mathrm{ClCHBr}_{2}$, chlorodibromomethane; $\mathrm{CHBr}_{3}$, bromoform; $\mathrm{THM}$, trihalomethane; NPTOX, nonpurgeable total organic halide; ND, not detected]

\begin{tabular}{ccccccc}
\hline $\begin{array}{c}\text { Reaction } \\
\text { time } \\
\text { (hours) }\end{array}$ & $\mathrm{CHCl}_{3}$ & $\mathrm{BrCHCl}_{2}$ & $\mathrm{ClCHBr}_{2}$ & $\mathrm{CHBr}_{3}$ & Total THM & NPTOX \\
\cline { 2 - 7 } & 50.7 & 5.73 & 0.14 & $\mathrm{ND}$ & 56.6 & 196 \\
0.00 & 195 & 21.4 & .57 & $\mathrm{ND}$ & 217 & 531 \\
2.00 & 246 & 24.2 & .71 & $\mathrm{ND}$ & 271 & 635 \\
6.00 & 289 & 25.4 & .75 & $\mathrm{ND}$ & 315 & 782 \\
12.00 & 368 & 29.0 & .87 & $\mathrm{ND}$ & 398 & 845 \\
24.00 & & & & & & \\
& & 30.5 & .93 & $\mathrm{ND}$ & 485 & 896 \\
48.00 & 454 & 32.8 & .98 & $\mathrm{ND}$ & 555 & 991 \\
72.00 & 521 & 33.8 & 1.01 & $\mathrm{ND}$ & 642 & 1,070 \\
126.0 & 607 & 34.0 & 1.07 & $\mathrm{ND}$ & 699 & 1,070 \\
168.0 & 664 & 34.2 & 1.03 & $\mathrm{ND}$ & 697 & 1,120 \\
168.0 & 662 & & & & & \\
\hline
\end{tabular}

Table 21.-Concentrations of the four trihalomethane compounds and the total trihalomethane and nonpurgeable total organic-halide concentrations as a function of reaction time for an initial pH of 7.50 and an initial free-chlorine concentration of 30.0 milligrams per liter, Missouri River 1.6 kilometers upstream from confluence with Mississippi River, September 30, 1991

$\left[\mu \mathrm{g} / \mathrm{L}\right.$, microgram per liter; $\mathrm{CHCl}_{3}$, chloroform; $\mathrm{BrCHCl}_{2}$, bromodichloromethane; $\mathrm{ClCHBr}_{2}$, chlorodibromomethane; $\mathrm{CHBr}_{3}$, bromoform; $\mathrm{THM}$, trihalomethane;

NPTOX, nonpurgeable total organic halide]

\begin{tabular}{ccccccc}
\hline $\begin{array}{c}\text { Reaction } \\
\text { time } \\
\text { (hours) }\end{array}$ & $\mathrm{CHCl}_{3}$ & $\mathrm{BrCHCl}_{2}$ & $\mathrm{ClCHBr}_{2}$ & $\mathrm{CHBr}_{3}$ & Total THM & NPTOX \\
\cline { 2 - 7 } & 7.77 & 4.50 & 2.30 & 0.14 & 14.7 & 44.2 \\
0.00 & 31.2 & 22.6 & 10.4 & .82 & 65.0 & 165 \\
2.00 & 43.0 & 28.5 & 13.0 & 1.04 & 85.5 & 202 \\
6.00 & 54.5 & 31.9 & 14.0 & 1.07 & 101 & 214 \\
12.00 & 77.1 & 38.4 & 15.4 & 1.15 & 132 & 258 \\
24.00 & & & & & & \\
& 100 & 43.7 & 16.5 & 1.21 & 161 & 281 \\
48.00 & 129 & 49.5 & 17.8 & 1.27 & 198 & 307 \\
72.00 & 155 & 52.7 & 18.0 & 1.22 & 227 & 329 \\
120.0 & 184 & 57.7 & 18.8 & 1.27 & 262 & 309 \\
168.0 & 182 & 57.5 & 18.9 & 1.29 & 260 & 351 \\
168.0 & & & & & &
\end{tabular}


Table 22.-Concentrations of the four trihalomethane compounds and the total trihaiomethane and nonpurgeabie total organic-halide concentrations as a function of reaction time for an initiai $\mathrm{pH}$ of 7.50 and an Initial free-chiorine concentration of 30.0 milligrams per liter, Mississippi River at St. Louis, Mo., September 30, 1991

[ $\mu \mathrm{g} / \mathrm{L}$, microgram per liter; $\mathrm{CHCl}_{3}$, chloroform; $\mathrm{BrCHCl}_{2}$, bromodichloromethane; $\mathrm{ClCHBr}_{2}$, chlorodibromomethane; $\mathrm{CHBr}_{3}$, bromoform; $\mathrm{THM}$, trihalomethane;

NPTOX, nonpurgeable total organic halide]

\begin{tabular}{ccccccc}
\hline $\begin{array}{c}\text { Reaction } \\
\text { time } \\
\text { (hours) }\end{array}$ & $\mathrm{CHCi}_{3}$ & $\mathrm{BrCHCi}_{2}$ & $\mathrm{CiCHBr}_{2}$ & $\mathrm{CHBr}_{3}$ & Totai THM & NPTOX \\
\cline { 2 - 7 } & 20.7 & 5.80 & 1.25 & 0.04 & 27.8 & 85.8 \\
0.00 & 79.7 & 28.4 & 5.47 & .13 & 114 & 234 \\
2.00 & 111 & 34.6 & 6.39 & .15 & 152 & 285 \\
6.07 & 128 & 37.6 & 6.78 & .16 & 173 & 376 \\
12.00 & 179 & 48.0 & 8.05 & .18 & 235 & 415 \\
24.02 & & & & & & \\
& 220 & 51.4 & 8.14 & .19 & 280 & 475 \\
48.00 & 254 & 55.0 & 8.63 & .18 & 318 & 502 \\
72.00 & 310 & 60.3 & 8.85 & .20 & 379 & 522 \\
125.0 & 350 & 64.5 & 9.07 & .21 & 424 & 549 \\
168.0 & 344 & 60.8 & 8.55 & .19 & 414 & 551 \\
168.0 & & & & & & \\
\hline
\end{tabular}

Tabie 23.-Concentrations of the four trihaiomethane compounds and the total trihalomethane and nonpurgeable total organic-halide concentrations as a function of reaction time for an initial pH of 7.50 and an initial free-chlorine concentration of 30.0 miiiigrams per iiter, Mississippi River at Cairo, iii., September 30, 1991

$\left[\mu \mathrm{g} / \mathrm{L}\right.$, microgram per liter; $\mathrm{CHCl}_{3}$, chloroform; $\mathrm{BrCHCl}_{2}$, bromodichloromethane; $\mathrm{ClCHBr}_{2}$, chlorodibromomethane; $\mathrm{CHBr}_{3}$, bromoform; $\mathrm{THM}$, trihalomethane;

NPTOX, nonpurgeable total organic halide]

\begin{tabular}{ccccccc}
\hline $\begin{array}{c}\text { Reaction } \\
\text { time } \\
\text { (hours) }\end{array}$ & $\mathrm{CHCl}_{3}$ & $\mathrm{BrCHCl}_{2}$ & $\mathrm{CiCHBr}_{2}$ & $\mathrm{CHBr}_{3}$ & Totai THM & NPTOX \\
\cline { 2 - 7 } & & & & & \\
\hline 0.00 & 28.8 & 7.86 & 0.78 & 0.02 & 37.5 & 117 \\
2.00 & 105 & 24.0 & 3.12 & .06 & 132 & 309 \\
6.00 & 141 & 30.6 & 4.11 & .07 & 176 & 406 \\
12.50 & 182 & 34.7 & 4.59 & .07 & 221 & 506 \\
24.00 & 226 & 39.3 & 4.86 & .07 & 270 & 551 \\
& & & & & & \\
48.00 & 282 & 43.5 & 5.15 & .07 & 331 & 564 \\
72.05 & 333 & 48.3 & 5.47 & .08 & 387 & 594 \\
120.0 & 408 & 55.0 & 6.29 & .08 & 469 & 677 \\
168.0 & 449 & 54.9 & 5.91 & .08 & 510 & 730 \\
168.0 & 437 & 54.2 & 5.85 & .08 & 497 & 723 \\
\hline
\end{tabular}


Table 24.--Concentrations of the four trihalomethane compounds and the total trihalomethane and nonpurgeabie total organic-halide concentrations as a function of reaction time for an initial pH of 7.50 and an inltiai free-chlorine concentration of 30.0 miligrams per iiter, Ohio River 1.6 kilometers upstream from confluence with Mississippi River, September 29, 1991

$\left[\mu \mathrm{g} / \mathrm{L}\right.$, microgram per liter; $\mathrm{CHCl}_{3}$, chloroform; $\mathrm{BrCHCl}_{2}$, bromodichloromethane; $\mathrm{ClCHBr}_{2}$, chlorodibromomethane; $\mathrm{CHBr}_{3}$, bromoform; $\mathrm{THM}$, trihalomethane; NPTOX, nonpurgeable total organic halide]

\begin{tabular}{ccccccc}
\hline $\begin{array}{c}\text { Reaction } \\
\text { time } \\
\text { (hours) }\end{array}$ & $\mathrm{CHCl}_{3}$ & $\mathrm{BrCHCi}_{2}$ & $\mathrm{ClCHBr}_{2}$ & $\mathrm{CHBr}_{3}$ & Total THM & NPTOX \\
\hline 0.00 & 12.1 & 3.36 & 0.92 & 0.04 & 16.4 & 64.8 \\
2.00 & 39.4 & 18.8 & 6.18 & .36 & 64.7 & 148 \\
6.00 & 55.1 & 24.6 & 7.83 & .45 & 88.0 & 203 \\
15.00 & 70.6 & 27.8 & 8.10 & .46 & 107 & 225 \\
24.00 & 84.7 & 30.3 & 8.59 & .48 & 124 & 202 \\
& & & & & & \\
48.00 & 117 & 34.9 & 9.27 & .51 & 162 & 206 \\
72.00 & 141 & 38.3 & 9.75 & .54 & 190 & 271 \\
122.0 & 172 & 41.3 & 10.0 & .53 & 224 & 252 \\
168.0 & 202 & 39.8 & 9.31 & .53 & 252 & 291 \\
168.0 & 197 & 39.8 & 9.61 & .55 & 247 & 270 \\
\hline
\end{tabular}

Table 25.-Concentrations of the four trihalomethane compounds and the total trihaiomethane and nonpurgeabie totai organic-halide concentrations as a function of reaction time for an initial pH of 7.50 and an initial free-chlorine concentration of 30.0 milligrams per iiter, Mississippi River at Memphis, Tenn., September 28, 1991

$\left[\mu \mathrm{g} / \mathrm{L}\right.$, microgram per liter; $\mathrm{CHCl}_{3}$, chloroform; $\mathrm{BrCHCl}_{2}$, bromodichloromethane; $\mathrm{ClCHBr}_{2}$, chlorodibromomethane; $\mathrm{CHBr}_{3}$, bromoform; $\mathrm{THM}$, trihalomethane; NPTOX, nonpurgeable total organic halide]

\begin{tabular}{ccccccc}
\hline $\begin{array}{c}\text { Reaction } \\
\text { time } \\
\text { (hours) }\end{array}$ & $\mathrm{CHCl}_{3}$ & $\mathrm{BrCHCl}_{2}$ & $\mathrm{ClCHBr}_{2}$ & $\mathrm{CHBr}_{3}$ & Totai THM & NPTOX \\
\hline 0.00 & 23.2 & 5.64 & 1.13 & 0.03 & 30.0 & 85.6 \\
2.00 & 76.7 & 25.8 & 4.50 & .09 & 107 & 219 \\
6.00 & 99.0 & 30.7 & 5.25 & .11 & 135 & 314 \\
12.00 & 120 & 33.7 & 5.78 & .13 & 160 & 324 \\
24.00 & 165 & 40.5 & 6.62 & .14 & 212 & 403 \\
& & & & & & \\
48.00 & 210 & 45.0 & 7.06 & .13 & 262 & 465 \\
72.07 & 243 & 47.6 & 7.38 & .14 & 298 & 509 \\
120.0 & 287 & 50.3 & 7.50 & .13 & 345 & 535 \\
168.0 & 322 & 51.8 & 7.40 & .15 & 381 & 515 \\
168.0 & 333 & 52.8 & 7.52 & .15 & 393 & 541 \\
\hline
\end{tabular}


Tabie 26.-Concentrations of the four trihaiomethane compounds and the totai trihaiomethane and nonpurgeabie totai organic-haiide concentrations as a function of reaction time for an initiai pH of 7.50 and an initiai free-chiorine concentration of 30.0 miliigrams per iiter, Mississippi River at Greenviile, Miss., September 27, 1991

$\left[\mu \mathrm{g} / \mathrm{L}\right.$, microgram per liter; $\mathrm{CHCl}_{3}$, chloroform; $\mathrm{BrCHCl}_{2}$, bromodichloromethane; $\mathrm{ClCHBr}_{2}$, chlorodibromomethane; $\mathrm{CHBr}_{3}$, bromoform; $\mathrm{THM}$, trihalomethane;

NPTOX, nonpurgeable total organic halide]

\begin{tabular}{ccccccc}
\hline $\begin{array}{c}\text { Reaction } \\
\text { time } \\
\text { (hours) }\end{array}$ & $\mathrm{CHCi}_{3}$ & $\mathrm{BrCHCi}_{2}$ & CiCHBr $_{2}$ & $\mathrm{CHBr}_{3}$ & Totai THM & NPTOX \\
\cline { 2 - 7 } & 18.1 & 4.33 & 1.02 & 0.04 & 23.5 & 62.7 \\
\hline 0.00 & 65.8 & 23.1 & 4.73 & .14 & 93.8 & 261 \\
2.00 & 85.2 & 30.3 & 6.29 & .18 & 122 & 242 \\
6.00 & 108 & 33.6 & 6.76 & .18 & 149 & 331 \\
12.00 & 143 & 38.5 & 7.24 & .19 & 189 & 384 \\
24.00 & & & & & & \\
& 187 & 44.4 & 8.02 & .20 & 240 & 439 \\
48.00 & 224 & 48.2 & 8.41 & .21 & 281 & 440 \\
72.00 & 267 & 52.0 & 8.79 & .21 & 328 & 415 \\
120.2 & 311 & 56.5 & 9.25 & .22 & 377 & 433 \\
168.0 & 307 & 56.4 & 9.20 & .22 & 373 & 463 \\
168.0 & & & & &
\end{tabular}

Tabie 27.-Concentrations of the four trihaiomethane compounds and the total trihaiomethane and nonpurgeable total organic-hailde concentrations as a function of reaction time for an initiai pH of $\mathbf{7 . 5 0}$ and an initial free-chlorine concentration of $\mathbf{3 0 . 0}$ miliigrams per liter, Mississippi River at Natchez, Miss., September 26, 1991

$\left[\mu \mathrm{g} / \mathrm{L}\right.$, microgram per liter; $\mathrm{CHCl}_{3}$, chloroform; $\mathrm{BrCHCl}_{2}$, bromodichloromethane; $\mathrm{ClCHBr}_{2}$, chlorodibromomethane; $\mathrm{CHBr}_{3}$, bromoform; $\mathrm{THM}$, trihalomethane; NPTOX, nonpurgeable total organic halide]

\begin{tabular}{ccccccc}
\hline $\begin{array}{c}\text { Reaction } \\
\text { time } \\
\text { (hours) }\end{array}$ & $\mathrm{CHCl}_{3}$ & $\mathrm{BrCHCl}_{2}$ & $\mathrm{CiCHBr}_{2}$ & $\mathrm{CHBr}_{3}$ & Totai THM & NPTOX \\
\hline 0.00 & 15.5 & 3.44 & 0.82 & 0.03 & 19.8 & 70.8 \\
2.00 & 59.2 & 19.8 & 4.70 & .18 & 83.9 & 191 \\
6.00 & 82.0 & 25.0 & 5.73 & .21 & 113 & 256 \\
12.00 & 97.9 & 27.7 & 6.09 & .22 & 132 & 302 \\
24.00 & 125 & 31.8 & 6.67 & .23 & 164 & 285 \\
& & & & & & \\
48.00 & 174 & 39.4 & 7.68 & .25 & 221 & 334 \\
72.00 & 193 & 40.3 & 7.81 & .25 & 241 & 348 \\
125.0 & 242 & 46.0 & 8.25 & .26 & 297 & 405 \\
168.0 & 278 & 50.7 & 8.91 & .29 & 338 & 406 \\
168.0 & 276 & 49.8 & 8.72 & .28 & 335 & 389 \\
\hline
\end{tabular}


Table 28.-Concentrations of the four trihalomethane compounds and the total trihalomethane and nonpurgeable total organic-halide concentrations as a function of reaction time for an Initial pH of 7.50 and an Initlal free-chlorine concentration of 30.0 milligrams per liter, MissIssippi River at Baton Rouge, La., September 26, 1991

$\left[\mu \mathrm{g} / \mathrm{L}\right.$, microgram per liter; $\mathrm{CHCl}_{3}$, chloroform; $\mathrm{BrCHCl}_{2}$, bromodichloromethane; $\mathrm{ClCHBr}_{2}$, chlorodibromomethane; $\mathrm{CHBr}_{3}$, bromoform; $\mathrm{THM}$, trihalomethane;

NPTOX, nonpurgeable total organic halide]

\begin{tabular}{ccccccc}
\hline $\begin{array}{c}\text { Reaction } \\
\text { time } \\
\text { (hours) }\end{array}$ & $\mathrm{CHCl}_{3}$ & $\mathrm{BrCHCl}_{2}$ & $\mathrm{ClCHBr}_{2}$ & $\mathrm{CHBr}_{3}$ & Total THM & NPTOX \\
\cline { 2 - 7 } & 13.1 & 2.92 & 0.80 & 0.05 & 16.9 & 94.7 \\
\hline 0.00 & 50.3 & 19.2 & 5.76 & .24 & 75.5 & 207 \\
2.00 & 70.9 & 24.4 & 6.96 & .30 & 103 & 235 \\
6.00 & 86.3 & 28.4 & 7.74 & .32 & 123 & 341 \\
12.00 & 112 & 33.1 & 8.47 & .35 & 154 & 384 \\
24.00 & & & & & & \\
& 140 & 35.8 & 8.58 & .33 & 185 & 338 \\
48.00 & 177 & 41.2 & 9.43 & .36 & 228 & 320 \\
72.00 & 220 & 45.2 & 9.75 & .38 & 275 & 405 \\
127.0 & 230 & 46.8 & 9.96 & .41 & 287 & 398 \\
168.0 & 239 & 47.4 & 10.1 & .41 & 297 & 369 \\
168.0 & & &
\end{tabular}

Table 29.-Concentrations of the four trihalomethane compounds and the total trihalomethane and nonpurgeable total organic-halide concentrations as a function of reaction time for an initial pH of 7.50 and an initial free-chlorine concentration of 30.0 milligrams per liter, Mississippi River at New Orleans, La., September 25, 1991

$\left[\dot{\mu} \mathrm{g} / \mathrm{L}\right.$, microgram per liter; $\mathrm{CHCl}_{3}$, chloroform; $\mathrm{BrCHCl}_{2}$, bromodichloromethane; $\mathrm{ClCHBr}_{2}$, chlorodibromomethane; $\mathrm{CHBr}_{3}$, bromoform; $\mathrm{THM}$, trihalomethane; NPTOX, nonpurgeable total organic halide]

\begin{tabular}{ccccccc}
\hline $\begin{array}{c}\text { Reaction } \\
\text { time } \\
\text { (hours) }\end{array}$ & $\mathrm{CHCl}_{3}$ & $\mathrm{BrCHCl}_{2}$ & $\mathrm{ClCHBr}_{2}$ & $\mathrm{CHBr}_{3}$ & Total THM & NPTOX \\
\cline { 2 - 7 } & 16.8 & 4.29 & 1.17 & 0.08 & 22.3 & 53.8 \\
\hline 0.00 & 59.2 & 22.5 & 6.51 & .34 & 88.6 & 217 \\
2.00 & 80.3 & 26.9 & 7.42 & .37 & 115 & 279 \\
6.00 & 101 & 32.0 & 8.76 & .43 & 142 & 264 \\
12.00 & 125 & 33.2 & 8.43 & .38 & 167 & 339 \\
24.00 & & & & & & \\
& 158 & 38.4 & 9.04 & .40 & 206 & 304 \\
48.00 & 193 & 47.4 & 10.6 & .48 & 251 & 313 \\
72.00 & 233 & 52.7 & 11.2 & .52 & 297 & 391 \\
125.0 & 251 & 53.7 & 11.0 & .49 & 316 & 439 \\
168.0 & 255 & 54.9 & 11.4 & .50 & 322 & 445 \\
168.0 & & & & & &
\end{tabular}




\section{Ancillary Data}

\section{Values of $\mathrm{pH}$ as a Function of Reaction Time}

Values of $\mathrm{pH}$ as a function of reaction time for the different combinations of initial $\mathrm{pH}$ and initial free-chlorine concentration are listed in tables 30-43.

Table 30.-Variation of $\mathrm{pH}$ with reaction time for different combinations of initial pH and initial free-chlorine concentration, Mississippi River at Minneapolis, Minn., October 7, 1991

[CL, initial free-chlorine concentration; $\mathrm{mg} / \mathrm{L}$, milligram per liter]

\begin{tabular}{|c|c|c|c|c|c|}
\hline \multicolumn{2}{|c|}{$C L=15.0 \mathrm{mg} / \mathrm{L}$} & \multicolumn{2}{|c|}{$C L=30.0 \mathrm{mg} / \mathrm{L}$} & \multicolumn{2}{|c|}{$C L=50.0 \mathrm{mg} / \mathrm{L}$} \\
\hline $\begin{array}{l}\text { Reaction } \\
\text { time } \\
\text { (hours) }\end{array}$ & pH & $\begin{array}{c}\text { Reaction } \\
\text { time } \\
\text { (hours) }\end{array}$ & pH & $\begin{array}{c}\text { Reaction } \\
\text { time } \\
\text { (hours) }\end{array}$ & pH \\
\hline \multicolumn{6}{|c|}{ Initial $\mathrm{pH}=5.50$} \\
\hline 0.00 & 5.50 & 0.00 & 5.50 & 0.00 & 5.50 \\
\hline 1.48 & 6.54 & 1.38 & 6.59 & 1.30 & 6.73 \\
\hline 21.78 & 6.52 & 21.68 & 6.55 & 21.60 & 6.64 \\
\hline 46.22 & 6.56 & 46.05 & 6.55 & 45.90 & 6.63 \\
\hline 78.98 & 6.60 & 78.92 & 6.61 & 78.83 & 6.62 \\
\hline 124.6 & 6.68 & 124.5 & 6.75 & 124.4 & 6.65 \\
\hline 170.3 & 6.87 & 170.2 & 6.78 & 170.2 & 6.67 \\
\hline \multicolumn{6}{|c|}{ Initial $\mathbf{p H}=\mathbf{7 . 5 0}$} \\
\hline .00 & 7.50 & .00 & 7.50 & .00 & 7.50 \\
\hline 2.52 & 8.22 & 2.43 & 8.16 & 2.32 & 8.49 \\
\hline 22.82 & 8.09 & 22.73 & 8.00 & 22.62 & 8.34 \\
\hline 47.00 & 8.10 & 46.85 & 7.97 & 46.67 & 8.37 \\
\hline 80.05 & 8.12 & 79.98 & 8.06 & 79.87 & 8.42 \\
\hline 125.7 & 8.18 & 125.6 & 8.16 & 125.5 & 8.51 \\
\hline 171.4 & 8.20 & 171.3 & 8.27 & 171.2 & 8.56 \\
\hline \multicolumn{6}{|c|}{ Initial pH $=10.00$} \\
\hline .00 & 10.00 & .00 & 10.00 & .00 & 10.00 \\
\hline .90 & 9.79 & .82 & 9.87 & .73 & 9.91 \\
\hline 21.18 & 9.63 & 21.10 & 9.72 & 21.02 & 9.75 \\
\hline 45.17 & 9.60 & 45.02 & 9.71 & 44.87 & 9.74 \\
\hline 78.43 & 9.57 & 78.37 & 9.67 & 78.28 & 9.70 \\
\hline 124.0 & 9.56 & 124.0 & 9.66 & 123.9 & 9.67 \\
\hline 169.8 & 9.53 & 169.7 & 9.64 & 169.6 & 9.64 \\
\hline
\end{tabular}


Table 31.-Variation of $\mathrm{pH}$ with reaction time for different combinations of initial $\mathrm{pH}$ and initial free-chlorine concentration, Mississippi River at LaCrosse, Wis., October 4, 1991

[CL, initial free-chlorine concentration; $\mathrm{mg} / \mathrm{L}$, milligram per liter]

\begin{tabular}{|c|c|c|c|c|c|}
\hline \multicolumn{2}{|c|}{$C L=15.0 \mathrm{mg} / \mathrm{L}$} & \multicolumn{2}{|c|}{$C L=30.0 \mathrm{mg} / \mathrm{L}$} & \multicolumn{2}{|c|}{$\mathrm{CL}=50.0 \mathrm{mg} / \mathrm{L}$} \\
\hline $\begin{array}{c}\text { Reaction } \\
\text { time } \\
\text { (hours) }\end{array}$ & pH & $\begin{array}{c}\text { Reaction } \\
\text { time } \\
\text { (hours) }\end{array}$ & pH & $\begin{array}{c}\text { Reaction } \\
\text { time } \\
\text { (hours) }\end{array}$ & pH \\
\hline \multicolumn{6}{|c|}{ Initial pH $=\mathbf{5 . 5 0}$} \\
\hline 0.00 & 5.50 & 0.00 & 5.50 & 0.00 & 5.50 \\
\hline 1.43 & 6.58 & 1.35 & 6.64 & 1.23 & 6.93 \\
\hline 26.42 & 6.53 & 26.32 & 6.53 & 26.20 & 6.76 \\
\hline 54.73 & 6.57 & 54.55 & 6.55 & 54.37 & 6.76 \\
\hline 78.90 & 6.68 & 78.72 & 6.62 & 78.53 & 6.79 \\
\hline 121.0 & 6.77 & 120.8 & 6.75 & 120.6 & 6.97 \\
\hline 170.5 & 7.08 & 170.3 & 6.90 & 170.1 & 6.99 \\
\hline \multicolumn{6}{|c|}{ Initial $\mathrm{pH}=\mathbf{7 . 5 0}$} \\
\hline .00 & 7.50 & .00 & 7.50 & .00 & 7.50 \\
\hline 2.57 & 8.27 & 2.32 & 8.27 & 2.50 & 8.56 \\
\hline 27.52 & 8.05 & 27.27 & 8.03 & 27.45 & 8.34 \\
\hline 55.60 & 8.06 & 55.25 & 7.99 & 55.37 & 8.29 \\
\hline 79.75 & 8.09 & 79.43 & 7.98 & 79.55 & 8.28 \\
\hline 121.8 & 8.10 & 121.5 & 8.19 & 121.6 & 8.27 \\
\hline 171.3 & 8.20 & 171.0 & 8.23 & 171.1 & 8.31 \\
\hline \multicolumn{6}{|c|}{ Initial $\mathrm{pH}=10.00$} \\
\hline .00 & 10.00 & .00 & 10.00 & .00 & 10.00 \\
\hline .83 & 9.97 & .73 & 10.06 & .65 & 10.08 \\
\hline 25.78 & 9.65 & 25.68 & 9.71 & 25.60 & 9.69 \\
\hline 53.63 & 9.63 & 53.45 & 9.69 & 53.30 & 9.60 \\
\hline 77.82 & 9.59 & 77.65 & 9.65 & 77.50 & 9.54 \\
\hline 119.8 & 9.57 & 119.7 & 9.62 & 119.5 & 9.45 \\
\hline 169.4 & 9.57 & 169.2 & 9.62 & 169.1 & 9.38 \\
\hline
\end{tabular}


Table 32.-Variation of $\mathrm{pH}$ with reaction time for different combinations of initial $\mathrm{pH}$ and Inltial free-chlorine concentration, MlssissIppl River at Dubuque, lowa, October 3, 1991

[CL, initial free-chlorine concentration; $\mathrm{mg} / \mathrm{L}$, milligram per liter]

\begin{tabular}{|c|c|c|c|c|c|}
\hline \multicolumn{2}{|c|}{$C L=15.0 \mathrm{mg} / \mathrm{L}$} & \multicolumn{2}{|c|}{$C L=30.0 \mathrm{mg} / \mathrm{L}$} & \multicolumn{2}{|c|}{$C L=50.0 \mathrm{mg} / \mathrm{L}$} \\
\hline $\begin{array}{l}\text { Reaction } \\
\text { time } \\
\text { (hours) }\end{array}$ & $\mathbf{p H}$ & $\begin{array}{c}\text { Reaction } \\
\text { time } \\
\text { (hours) }\end{array}$ & pH & $\begin{array}{c}\text { Reaction } \\
\text { time } \\
\text { (hours) }\end{array}$ & pH \\
\hline \multicolumn{6}{|c|}{ Initial $\mathrm{pH}=\mathbf{5 . 5 0}$} \\
\hline 0.00 & 5.50 & 0.00 & 5.50 & 0.00 & 5.50 \\
\hline 1.78 & 6.65 & 1.62 & 6.74 & 1.45 & 7.04 \\
\hline 31.37 & 6.55 & 31.20 & 6.60 & 31.03 & 6.87 \\
\hline 53.73 & 6.58 & 53.57 & 6.62 & 53.35 & 6.87 \\
\hline 78.83 & 6.63 & 78.65 & 6.66 & 78.48 & 6.88 \\
\hline 124.0 & 6.70 & 123.9 & 6.81 & 123.7 & 6.92 \\
\hline 170.4 & 6.73 & 170.2 & 6.80 & 170.1 & 6.93 \\
\hline \multicolumn{6}{|c|}{ Initial $\mathbf{p H}=\mathbf{7 . 5 0}$} \\
\hline .00 & 7.50 & .00 & 7.50 & .00 & 7.50 \\
\hline 2.67 & 8.27 & 2.33 & 8.33 & 2.47 & 8.59 \\
\hline 32.25 & 8.15 & 31.92 & 8.19 & 32.03 & 8.41 \\
\hline 54.58 & 8.12 & 54.25 & 8.20 & 54.38 & 8.37 \\
\hline 79.72 & 8.12 & 79.38 & 8.24 & 79.52 & 8.36 \\
\hline 124.9 & 8.12 & 124.6 & 8.32 & 124.7 & 8.41 \\
\hline 171.3 & 8.12 & 171.0 & 8.39 & 171.1 & 8.38 \\
\hline \multicolumn{6}{|c|}{ Initial pH = 10.00} \\
\hline .00 & 10.00 & .00 & 10.00 & .00 & 10.00 \\
\hline .77 & 9.99 & .60 & 10.03 & .43 & 10.05 \\
\hline 30.32 & 9.70 & 30.13 & 9.75 & 29.97 & 9.75 \\
\hline 52.63 & 9.69 & 52.47 & 9.73 & 52.30 & 9.71 \\
\hline 77.82 & 9.65 & 77.65 & 9.70 & 77.47 & 9.66 \\
\hline 123.0 & 9.64 & 122.8 & 9.69 & 122.7 & 9.58 \\
\hline 169.4 & 9.63 & 169.2 & 9.67 & 169.1 & 9.53 \\
\hline
\end{tabular}


Table 33.-Variation of pH with reaction time for different combinations of initial pH and initial free-chlorine concentration, Mississippi River at Davenport, lowa, October 2, 1991

[CL, initial free-chlorine concentration; $\mathrm{mg} / \mathrm{L}$, milligram per liter]

\begin{tabular}{|c|c|c|c|c|c|}
\hline \multicolumn{2}{|c|}{$C L=15.0 \mathrm{mg} / \mathrm{L}$} & \multicolumn{2}{|c|}{$C L=30.0 \mathrm{mg} / \mathrm{L}$} & \multicolumn{2}{|c|}{$C L=50.0 \mathrm{mg} / \mathrm{L}$} \\
\hline $\begin{array}{c}\text { Reaction } \\
\text { time } \\
\text { (hours) }\end{array}$ & pH & $\begin{array}{c}\text { Reaction } \\
\text { time } \\
\text { (hours) }\end{array}$ & pH & $\begin{array}{c}\text { Reaction } \\
\text { time } \\
\text { (hours) }\end{array}$ & pH \\
\hline \multicolumn{6}{|c|}{ Initial $\mathbf{p H}=5.50$} \\
\hline 0.00 & 5.50 & 0.00 & 5.50 & 0.00 & 5.50 \\
\hline 1.42 & 6.63 & 1.32 & 6.70 & 1.22 & 6.93 \\
\hline 27.07 & 6.54 & 26.90 & 6.59 & 26.73 & 6.76 \\
\hline 55.35 & 6.57 & 55.18 & 6.62 & 54.98 & 6.79 \\
\hline 79.82 & 6.64 & 79.63 & 6.69 & 79.47 & 6.85 \\
\hline 120.0 & 6.65 & 119.8 & 6.68 & 119.6 & 6.83 \\
\hline 170.4 & 6.74 & 170.2 & 6.72 & 170.0 & 6.86 \\
\hline \multicolumn{6}{|c|}{ Initial $\mathrm{pH}=\mathbf{7 . 5 0}$} \\
\hline .00 & 7.50 & .00 & 7.50 & .00 & 7.50 \\
\hline 2.57 & 8.30 & 2.32 & 8.34 & 2.50 & 8.62 \\
\hline 27.97 & 8.11 & 27.65 & 8.17 & 27.77 & 8.42 \\
\hline 56.25 & 8.09 & 55.93 & 8.13 & 56.05 & 8.38 \\
\hline 80.70 & 8.13 & 80.37 & 8.11 & 80.48 & 8.40 \\
\hline 120.9 & 8.07 & 120.6 & 8.03 & 120.7 & 8.32 \\
\hline 171.3 & 8.06 & 171.0 & 8.05 & 171.0 & 8.30 \\
\hline \multicolumn{6}{|c|}{ Initial $\mathrm{pH}=10.00$} \\
\hline .00 & 10.00 & .00 & 10.00 & .00 & 10.00 \\
\hline .80 & 10.03 & .70 & 10.05 & .63 & 10.07 \\
\hline 25.97 & 9.71 & 25.80 & 9.77 & 25.65 & 9.79 \\
\hline 54.27 & 9.69 & 54.10 & 9.73 & 53.90 & 9.77 \\
\hline 78.68 & 9.71 & 78.48 & 9.74 & 78.33 & 9.77 \\
\hline 118.9 & 9.66 & 118.8 & 9.70 & 118.6 & 9.73 \\
\hline 169.3 & 9.65 & 169.1 & 9.67 & 169.0 & 9.71 \\
\hline
\end{tabular}


Table 34.-Variation of pH with reaction time for different combinations of initial pH and initial free-chlorine concentration, Mississippi River at Quincy, III., October 1, 1991

[CL, initial free-chlorine concentration; $\mathrm{mg} / \mathrm{L}$, milligram per liter]

\begin{tabular}{|c|c|c|c|c|c|}
\hline \multicolumn{2}{|c|}{$C L=15.0 \mathrm{mg} / \mathrm{L}$} & \multicolumn{2}{|c|}{$C L=30.0 \mathrm{mg} / \mathrm{L}$} & \multicolumn{2}{|c|}{$C L=50.0 \mathrm{mg} / \mathrm{L}$} \\
\hline $\begin{array}{l}\text { Reaction } \\
\text { time } \\
\text { (hours) }\end{array}$ & pH & $\begin{array}{c}\text { Reaction } \\
\text { time } \\
\text { (hours) }\end{array}$ & pH & $\begin{array}{c}\text { Reaction } \\
\text { time } \\
\text { (hours) }\end{array}$ & pH \\
\hline \multicolumn{6}{|c|}{ Initial $\mathbf{p H}=5.50$} \\
\hline 0.00 & 5.50 & 0.00 & 5.50 & 0.00 & 5.50 \\
\hline 1.78 & 6.40 & 1.62 & 6.53 & 1.42 & 6.80 \\
\hline 31.00 & 6.40 & 30.82 & 6.50 & 30.63 & 6.72 \\
\hline 54.82 & 6.47 & 54.65 & 6.54 & 54.47 & 6.74 \\
\hline 79.50 & 6.52 & 79.33 & 6.58 & 79.13 & 6.78 \\
\hline 124.9 & 6.59 & 124.7 & 6.64 & 124.6 & 6.82 \\
\hline 170.5 & 6.61 & 170.4 & 6.68 & 170.2 & 6.84 \\
\hline \multicolumn{6}{|c|}{ Initial $\mathrm{pH}=\mathbf{7 . 5 0}$} \\
\hline .00 & 7.50 & .00 & 7.50 & .00 & 7.50 \\
\hline 2.68 & 8.18 & 2.33 & 8.22 & 2.40 & 8.49 \\
\hline 31.83 & 8.10 & 31.48 & 8.10 & 31.62 & 8.39 \\
\hline 55.73 & 8.08 & 55.40 & 8.07 & 55.53 & 8.35 \\
\hline 80.40 & 8.08 & 80.07 & 8.08 & 80.18 & 8.33 \\
\hline 125.8 & 8.16 & 125.5 & 8.10 & 125.6 & 8.31 \\
\hline 171.4 & 8.19 & 171.1 & 8.15 & 171.2 & 8.31 \\
\hline \multicolumn{6}{|c|}{ Initial $\mathrm{pH}=10.00$} \\
\hline .00 & 10.00 & .00 & 10.00 & .00 & 10.00 \\
\hline .73 & 9.90 & .52 & 9.95 & .35 & 9.94 \\
\hline 29.83 & 9.66 & 29.63 & 9.70 & 29.47 & 9.68 \\
\hline 53.73 & 9.64 & 53.58 & 9.67 & 53.42 & 9.65 \\
\hline 78.38 & 9.61 & 78.23 & 9.65 & 78.07 & 9.64 \\
\hline 123.9 & 9.60 & 123.7 & 9.64 & 123.6 & 9.63 \\
\hline 169.5 & 9.62 & 169.3 & 9.65 & 169.1 & 9.64 \\
\hline
\end{tabular}


Tabie 35.-Variation of $\mathrm{pH}$ with reaction time for different combinations of initiai $\mathrm{pH}$ and initiai free-chiorine concentration, Mlssouri River 1.6 kiiometers upstream from confiuence with Mississippi River, September 30, 1991

[CL, initial free-chlorine concentration; $\mathrm{mg} / \mathrm{L}$, milligram per liter]

\begin{tabular}{|c|c|c|c|c|c|}
\hline \multicolumn{2}{|c|}{$C L=15.0 \mathrm{mg} / \mathrm{L}$} & \multicolumn{2}{|c|}{$C L=30.0 \mathrm{mg} / \mathrm{L}$} & \multicolumn{2}{|c|}{$C L=50.0 \mathrm{mg} / \mathrm{L}$} \\
\hline $\begin{array}{c}\text { Reaction } \\
\text { time } \\
\text { (hours) }\end{array}$ & pH & $\begin{array}{c}\text { Reaction } \\
\text { time } \\
\text { (hours) }\end{array}$ & pH & $\begin{array}{c}\text { Reaction } \\
\text { time } \\
\text { (hours) }\end{array}$ & pH \\
\hline \multicolumn{6}{|c|}{ Initial pH $=5.50$} \\
\hline 0.00 & 5.50 & 0.00 & 5.50 & 0.00 & 5.50 \\
\hline 1.53 & 6.39 & 1.43 & 6.56 & 1.35 & 6.84 \\
\hline 27.07 & 6.40 & 26.90 & 6.54 & 26.73 & 6.79 \\
\hline 55.18 & 6.51 & 55.02 & 6.62 & 54.83 & 6.86 \\
\hline 77.42 & 6.57 & 77.25 & 6.68 & 77.05 & 6.91 \\
\hline 120.0 & 6.59 & 119.9 & 6.70 & 119.7 & 6.93 \\
\hline 170.3 & 6.67 & 170.2 & 6.75 & 170.0 & 7.00 \\
\hline \multicolumn{6}{|c|}{ Initial pH $=\mathbf{7 . 5 0}$} \\
\hline .00 & 7.50 & .00 & 7.50 & .00 & 7.50 \\
\hline 2.65 & 8.23 & 2.38 & 8.30 & 2.58 & 8.58 \\
\hline 27.95 & 8.13 & 27.62 & 8.18 & 27.75 & 8.47 \\
\hline 56.00 & 8.16 & 55.67 & 8.23 & 55.80 & 8.48 \\
\hline 78.27 & 8.16 & 77.92 & 8.23 & 78.05 & 8.48 \\
\hline 120.9 & 8.13 & 120.5 & 8.22 & 120.6 & 8.46 \\
\hline 171.2 & 8.11 & 170.8 & 8.31 & 171.0 & 8.45 \\
\hline \multicolumn{6}{|c|}{ Initial pH = 10.00} \\
\hline .00 & 10.00 & .00 & 10.00 & .00 & 10.00 \\
\hline .97 & 9.79 & .88 & 9.83 & .80 & 9.86 \\
\hline 26.03 & 9.57 & 25.88 & 9.63 & 25.73 & 9.66 \\
\hline 54.12 & 9.58 & 53.97 & 9.63 & 53.82 & 9.66 \\
\hline 76.35 & 9.55 & 76.20 & 9.61 & 76.03 & 9.65 \\
\hline 118.9 & 9.54 & 118.8 & 9.57 & 118.6 & 9.63 \\
\hline 169.3 & 9.51 & 169.1 & 9.56 & 169.0 & 9.60 \\
\hline
\end{tabular}


Table 36.-Varlation of pH with reactlon time for different comblnations of InItlal pH and initial free-chlorine concentration, Mississippi River at St. Louis, Mo., September 30, 1991

[CL, initial free-chlorine concentration; $\mathrm{mg} / \mathrm{L}$, milligram per liter]

\begin{tabular}{|c|c|c|c|c|c|}
\hline \multicolumn{2}{|c|}{$C L=15.0 \mathrm{mg} / \mathrm{L}$} & \multicolumn{2}{|c|}{$C L=30.0 \mathrm{mg} / \mathrm{L}$} & \multicolumn{2}{|c|}{$C L=50.0 \mathrm{mg} / \mathrm{L}$} \\
\hline $\begin{array}{c}\text { Reaction } \\
\text { time } \\
\text { (hours) }\end{array}$ & pH & $\begin{array}{c}\text { Reaction } \\
\text { time } \\
\text { (hours) }\end{array}$ & pH & $\begin{array}{c}\text { Reaction } \\
\text { time } \\
\text { (hours) }\end{array}$ & pH \\
\hline \multicolumn{6}{|c|}{ Initial $\mathbf{p H}=\mathbf{5 . 5 0}$} \\
\hline 0.00 & 5.50 & 0.00 & 5.50 & 0.00 & 5.50 \\
\hline 1.78 & 6.44 & 1.63 & 6.55 & 1.45 & 6.81 \\
\hline 30.58 & 6.48 & 30.50 & 6.53 & 30.38 & 6.78 \\
\hline 53.08 & 6.61 & 53.00 & 6.58 & 52.88 & 6.83 \\
\hline 79.03 & 6.71 & 78.95 & 6.62 & 78.83 & 6.86 \\
\hline 123.8 & 6.88 & 123.7 & 6.66 & 123.6 & 6.88 \\
\hline 170.8 & 6.97 & 170.6 & 6.67 & 170.4 & 6.90 \\
\hline \multicolumn{6}{|c|}{ Initial $\mathrm{pH}=\mathbf{7 . 5 0}$} \\
\hline .00 & 7.50 & .00 & 7.50 & .00 & 7.50 \\
\hline 2.67 & 8.17 & 2.33 & 8.25 & 2.47 & 8.50 \\
\hline 31.70 & 8.10 & 31.47 & 8.18 & 31.67 & 8.44 \\
\hline 54.25 & 8.12 & 53.98 & 8.18 & 54.18 & 8.44 \\
\hline 80.15 & 8.09 & 79.90 & 8.17 & 80.10 & 8.42 \\
\hline 124.9 & 8.12 & 124.6 & 8.17 & 124.8 & 8.42 \\
\hline 171.6 & 8.12 & 171.2 & 8.16 & 171.4 & 8.40 \\
\hline \multicolumn{6}{|c|}{ Initial $\mathrm{pH}=10.00$} \\
\hline .00 & 10.00 & .00 & 10.00 & .00 & 10.00 \\
\hline .78 & 9.79 & .62 & 9.81 & .45 & 9.85 \\
\hline 31.07 & 9.64 & 30.98 & 9.66 & 30.93 & 9.70 \\
\hline 52.58 & 9.62 & 52.48 & 9.65 & 52.40 & 9.68 \\
\hline 78.50 & 9.59 & 78.40 & 9.63 & 78.32 & 9.65 \\
\hline 123.2 & 9.58 & 123.2 & 9.62 & 123.1 & 9.62 \\
\hline 169.7 & 9.55 & 169.5 & 9.60 & 169.4 & 9.60 \\
\hline
\end{tabular}


Table 37.-Variation of $\mathrm{pH}$ with reaction time for different combinations of initial $\mathrm{pH}$ and initial free-chlorine concentration, Mississippi River at Cairo, III., September 30, 1991

[CL, initial free-chlorine concentration; $\mathrm{mg} / \mathrm{L}$, milligram per liter]

\begin{tabular}{|c|c|c|c|c|c|}
\hline \multicolumn{2}{|c|}{$C L=15.0 \mathrm{mg} / \mathrm{L}$} & \multicolumn{2}{|c|}{$C L=30.0 \mathrm{mg} / \mathrm{L}$} & \multicolumn{2}{|c|}{$C L=50.0 \mathrm{mg} / \mathrm{L}$} \\
\hline $\begin{array}{c}\text { Reaction } \\
\text { time } \\
\text { (hours) }\end{array}$ & pH & $\begin{array}{c}\text { Reaction } \\
\text { time } \\
\text { (hours) }\end{array}$ & pH & $\begin{array}{c}\text { Reaction } \\
\text { time } \\
\text { (hours) }\end{array}$ & pH \\
\hline \multicolumn{6}{|c|}{ Initial $\mathrm{pH}=\mathbf{5 . 5 0}$} \\
\hline 0.00 & 5.50 & 0.00 & 5.50 & 0.00 & 5.50 \\
\hline 1.82 & 6.45 & 1.65 & 6.56 & 1.50 & 6.82 \\
\hline 27.10 & 6.41 & 27.00 & 6.51 & 26.92 & 6.74 \\
\hline 54.95 & 6.44 & 54.78 & 6.54 & 54.63 & 6.79 \\
\hline 77.08 & 6.48 & 76.90 & 6.58 & 76.75 & 6.84 \\
\hline 119.9 & 6.53 & 119.7 & 6.66 & 119.6 & 6.88 \\
\hline 170.1 & 6.58 & 169.9 & 6.70 & 169.8 & 6.99 \\
\hline \multicolumn{6}{|c|}{ Initial $\mathrm{pH}=\mathbf{7 . 5 0}$} \\
\hline .00 & 7.50 & .00 & 7.50 & .00 & 7.50 \\
\hline 2.98 & 8.19 & 2.40 & 8.25 & 2.55 & 8.50 \\
\hline 28.48 & 8.18 & 27.97 & 8.14 & 28.18 & 8.41 \\
\hline 56.13 & 8.29 & 55.55 & 8.12 & 55.70 & 8.37 \\
\hline 78.23 & 8.35 & 77.62 & 8.13 & 77.67 & 8.38 \\
\hline 121.0 & 8.45 & 120.4 & 8.13 & 120.6 & 8.37 \\
\hline 171.2 & 8.55 & 170.6 & 8.13 & 170.8 & 8.37 \\
\hline \multicolumn{6}{|c|}{ Initial $\mathbf{p H}=10.00$} \\
\hline .00 & 10.00 & .00 & 10.00 & .00 & 10.00 \\
\hline .75 & 9.84 & .60 & 9.88 & .43 & 9.88 \\
\hline 26.47 & 9.70 & 26.38 & 9.73 & 26.28 & 9.74 \\
\hline 53.90 & 9.66 & 53.75 & 9.69 & 53.57 & 9.72 \\
\hline 75.85 & 9.67 & 75.70 & 9.68 & 75.47 & 9.72 \\
\hline 118.8 & 9.63 & 118.7 & 9.67 & 118.5 & 9.71 \\
\hline 169.0 & 9.62 & 168.9 & 9.64 & 168.7 & 9.69 \\
\hline
\end{tabular}


Table 38.-Variation of $\mathrm{pH}$ with reaction time for different combinations of initial pH and initial free-chlorine concentration, Ohio River 1.6 kilometers upstream from confluence with Mississippi River, September 29, 1991

[CL, initial free-chlorine concentration; $\mathrm{mg} / \mathrm{L}$, milligram per liter]

\begin{tabular}{|c|c|c|c|c|c|}
\hline \multicolumn{2}{|c|}{$C L=15.0 \mathrm{mg} / \mathrm{L}$} & \multicolumn{2}{|c|}{$\mathrm{CL}=30.0 \mathrm{mg} / \mathrm{L}$} & \multicolumn{2}{|c|}{$\mathrm{CL}=50.0 \mathrm{mg} / \mathrm{L}$} \\
\hline $\begin{array}{c}\text { Reaction } \\
\text { time } \\
\text { (hours) }\end{array}$ & pH & $\begin{array}{c}\text { Reaction } \\
\text { time } \\
\text { (hours) }\end{array}$ & pH & $\begin{array}{c}\text { Reaction } \\
\text { time } \\
\text { (hours) }\end{array}$ & pH \\
\hline \multicolumn{6}{|c|}{ Initial pH $=\mathbf{5 . 5 0}$} \\
\hline 0.00 & 5.50 & 0.00 & 5.50 & 0.00 & 5.50 \\
\hline 2.08 & 6.67 & 2.00 & 6.96 & 1.90 & 7.40 \\
\hline 31.33 & 6.74 & 31.18 & 6.94 & 31.02 & 7.35 \\
\hline 54.43 & 6.82 & 54.27 & 6.99 & 54.10 & 7.34 \\
\hline 78.63 & 6.93 & 78.47 & 7.00 & 78.30 & 7.34 \\
\hline 121.9 & 7.00 & 121.7 & 7.02 & 121.6 & 7.35 \\
\hline 171.4 & 7.06 & 171.2 & 7.10 & 171.1 & $7.37^{\circ}$ \\
\hline \multicolumn{6}{|c|}{ Initial $\mathbf{p H}=\mathbf{7 . 5 0}$} \\
\hline .00 & 7.50 & .00 & 7.50 & .00 & 7.50 \\
\hline 2.92 & 8.41 & 3.27 & 8.62 & 2.87 & 8.89 \\
\hline 31.93 & 8.32 & 32.18 & 8.54 & 31.72 & 8.82 \\
\hline 55.00 & 8.27 & 55.27 & 8.51 & 54.78 & 8.80 \\
\hline 79.23 & 8.26 & 79.50 & 8.46 & 79.03 & 8.77 \\
\hline 122.5 & 8.22 & 122.7 & 8.44 & 122.3 & 8.74 \\
\hline 172.0 & 8.23 & 172.3 & 8.51 & 171.8 & 8.72 \\
\hline \multicolumn{6}{|c|}{ Initial $\mathrm{pH}=10.00$} \\
\hline .00 & 10.00 & .00 & 10.00 & .00 & 10.00 \\
\hline .72 & 10.04 & .62 & 10.06 & .52 & 10.10 \\
\hline 29.50 & 9.82 & 29.32 & 9.82 & 29.15 & 9.91 \\
\hline 52.53 & 9.77 & 52.33 & 9.77 & 52.17 & 9.85 \\
\hline 76.82 & 9.71 & 76.65 & 9.72 & 76.48 & 9.81 \\
\hline 120.0 & 9.66 & 119.9 & 9.68 & 119.7 & 9.79 \\
\hline 169.6 & 9.61 & 169.4 & 9.66 & 169.2 & 9.76 \\
\hline
\end{tabular}


Table 39.-Variation of $\mathrm{pH}$ with reaction time for different combinations of initial pH and initial free-chlorine concentration, Mississippi River at Memphis, Tenn., September 28, 1991

[CL, initial free-chlorine concentration; $\mathrm{mg} / \mathrm{L}$, milligram per liter]

\begin{tabular}{|c|c|c|c|c|c|}
\hline \multicolumn{2}{|c|}{$C L=15.0 \mathrm{mg} / \mathrm{L}$} & \multicolumn{2}{|c|}{$C L=30.0 \mathrm{mg} / \mathrm{L}$} & \multicolumn{2}{|c|}{$C L=50.0 \mathrm{mg} / \mathrm{L}$} \\
\hline $\begin{array}{c}\text { Reaction } \\
\text { time } \\
\text { (hours) }\end{array}$ & pH & $\begin{array}{c}\text { Reaction } \\
\text { time } \\
\text { (hours) }\end{array}$ & pH & $\begin{array}{c}\text { Reaction } \\
\text { time } \\
\text { (hours) }\end{array}$ & pH \\
\hline \multicolumn{6}{|c|}{ Initial $\mathbf{p H}=5.50$} \\
\hline 0.00 & 5.50 & 0.00 & 5.50 & 0.00 & 5.50 \\
\hline 2.72 & 6.48 & 2.52 & 6.64 & 2.32 & 6.96 \\
\hline 23.12 & 6.57 & 22.95 & 6.71 & 22.77 & 6.99 \\
\hline 54.53 & 6.68 & 54.37 & 6.72 & 54.20 & 6.99 \\
\hline 76.47 & 6.94 & 76.30 & 6.78 & 76.12 & 7.01 \\
\hline 119.6 & 7.30 & 119.4 & 6.81 & 119.2 & 7.03 \\
\hline 172.8 & 7.35 & 172.7 & 6.86 & 172.6 & 7.08 \\
\hline \multicolumn{6}{|c|}{ Initial pH = 7.50 } \\
\hline .00 & 7.50 & .00 & 7.50 & .00 & 7.50 \\
\hline 3.23 & 8.16 & 3.47 & 8.21 & 3.02 & 8.53 \\
\hline 23.68 & 8.18 & 23.93 & 8.23 & 23.48 & 8.56 \\
\hline 55.13 & 8.14 & 55.38 & 8.18 & 54.93 & 8.50 \\
\hline 77.05 & 8.09 & 77.30 & 8.15 & 76.83 & 8.48 \\
\hline 120.2 & 8.08 & 120.4 & 8.13 & 120.0 & 8.46 \\
\hline 173.8 & 8.14 & 174.1 & 8.16 & 173.7 & 8.46 \\
\hline \multicolumn{6}{|c|}{ Initial $\mathbf{p H}=10.00$} \\
\hline .00 & 10.00 & .00 & 10.00 & .00 & 10.00 \\
\hline .72 & 9.94 & .55 & 9.96 & .40 & 10.02 \\
\hline 21.22 & 9.76 & 21.03 & 9.79 & 20.88 & 9.85 \\
\hline 52.65 & 9.74 & 52.48 & 9.75 & 52.33 & 9.79 \\
\hline 74.57 & 9.71 & 74.38 & 9.72 & 74.23 & 9.77 \\
\hline 117.7 & 9.68 & 117.5 & 9.71 & 117.4 & 9.76 \\
\hline 171.6 & 9.68 & 171.5 & 9.71 & 171.4 & 9.74 \\
\hline
\end{tabular}


Table 40.-Variation of pH with reaction time for different combinations of initial pH and initial free-chlorine concentration, Mississippi River at Greenville, Miss.,

September 27, 1991

[CL, initial free-chlorine concentration; $\mathrm{mg} / \mathrm{L}$, milligram per liter]

\begin{tabular}{|c|c|c|c|c|c|}
\hline \multicolumn{2}{|c|}{$C L=15.0 \mathrm{mg} / \mathrm{L}$} & \multicolumn{2}{|c|}{$\mathrm{CL}=30.0 \mathrm{mg} / \mathrm{L}$} & \multicolumn{2}{|c|}{$C L=50.0 \mathrm{mg} / \mathrm{L}$} \\
\hline $\begin{array}{c}\text { Reaction } \\
\text { time } \\
\text { (hours) }\end{array}$ & pH & $\begin{array}{c}\text { Reaction } \\
\text { time } \\
\text { (hours) }\end{array}$ & pH & $\begin{array}{c}\text { Reaction } \\
\text { time } \\
\text { (hours) }\end{array}$ & pH \\
\hline \multicolumn{6}{|c|}{ Initial pH $=5.50$} \\
\hline 0.00 & 5.50 & 0.00 & 5.50 & 0.00 & 5.50 \\
\hline 1.98 & 6.43 & 1.88 & 6.55 & 1.80 & 6.90 \\
\hline 29.12 & 6.40 & 29.02 & 6.53 & 28.93 & 6.85 \\
\hline 54.70 & 6.51 & 54.60 & 6.65 & 54.53 & 6.92 \\
\hline 77.65 & 6.55 & 77.55 & 6.68 & 77.47 & 6.99 \\
\hline 119.4 & 6.73 & 119.3 & 6.73 & 119.2 & 7.04 \\
\hline 171.4 & 6.79 & 171.3 & 6.92 & 171.2 & 7.10 \\
\hline \multicolumn{6}{|c|}{ Initial $\mathbf{p H}=\mathbf{7 . 5 0}$} \\
\hline .00 & 7.50 & .00 & 7.50 & .00 & 7.50 \\
\hline 2.88 & 8.25 & 3.20 & 8.31 & 2.82 & 8.63 \\
\hline 30.03 & 8.14 & 30.35 & 8.21 & 29.97 & 8.51 \\
\hline 55.63 & 8.13 & 55.98 & 8.21 & 55.62 & 8.50 \\
\hline 78.55 & 8.13 & 78.88 & 8.21 & 78.50 & 8.48 \\
\hline 120.3 & 8.14 & 120.7 & 8.22 & 120.3 & 8.47 \\
\hline 172.3 & 8.22 & 172.6 & 8.27 & 172.2 & 8.45 \\
\hline \multicolumn{6}{|c|}{ Initial $\mathbf{p H}=\mathbf{1 0 . 0 0}$} \\
\hline .00 & 10.00 & .00 & 10.00 & .00 & 10.00 \\
\hline .75 & 10.03 & .65 & 10.05 & .55 & 10.06 \\
\hline 27.87 & 9.67 & 27.77 & 9.71 & 27.67 & 9.72 \\
\hline 53.53 & 9.62 & 53.48 & 9.67 & 53.42 & 9.68 \\
\hline 76.40 & 9.60 & 76.30 & 9.65 & 76.20 & 9.68 \\
\hline 118.2 & 9.59 & 118.1 & 9.63 & 118.0 & 9.66 \\
\hline 170.2 & 9.60 & 170.0 & 9.64 & 170.0 & 9.67 \\
\hline
\end{tabular}


Table 41.-Variation of $\mathrm{pH}$ with reaction time for different combinations of initial $\mathrm{pH}$ and initial free-chlorine concentration, Mississippi River at Natchez, Miss., September 26, 1991

[CL, initial free-chlorine concentration; $\mathrm{mg} / \mathrm{L}$, milligram per liter]

\begin{tabular}{|c|c|c|c|c|c|}
\hline \multicolumn{2}{|c|}{$C L=15.0 \mathrm{mg} / \mathrm{L}$} & \multicolumn{2}{|c|}{$C L=30.0 \mathrm{mg} / \mathrm{L}$} & \multicolumn{2}{|c|}{$\mathrm{CL}=50.0 \mathrm{mg} / \mathrm{L}$} \\
\hline $\begin{array}{c}\text { Reaction } \\
\text { time } \\
\text { (hours) }\end{array}$ & pH & $\begin{array}{c}\text { Reaction } \\
\text { time } \\
\text { (hours) }\end{array}$ & pH & $\begin{array}{c}\text { Reaction } \\
\text { time } \\
\text { (hours) }\end{array}$ & pH \\
\hline \multicolumn{6}{|c|}{ Initial $\mathrm{pH}=\mathbf{5 . 5 0}$} \\
\hline 0.00 & 5.50 & 0.00 & 5.50 & 0.00 & 5.50 \\
\hline 4.25 & 6.45 & 4.17 & 6.73 & 4.07 & 6.98 \\
\hline 30.63 & 6.50 & 30.55 & 6.83 & 30.45 & 6.95 \\
\hline 52.65 & 6.55 & 52.57 & 6.95 & 52.47 & 6.98 \\
\hline 77.98 & 6.58 & 77.90 & 7.07 & 77.83 & 7.01 \\
\hline 123.6 & 6.66 & 123.6 & 7.30 & 123.4 & 7.06 \\
\hline 146.8 & 6.79 & 146.7 & 7.42 & 146.5 & 7.16 \\
\hline 173.0 & 6.77 & 172.9 & 7.43 & 172.8 & 7.15 \\
\hline \multicolumn{6}{|c|}{ Initial pH = 7.50} \\
\hline .00 & 7.50 & .00 & 7.50 & .00 & 7.50 \\
\hline 5.23 & 8.28 & 5.65 & 8.37 & 5.20 & 8.63 \\
\hline 31.53 & 8.17 & 31.95 & 8.27 & 31.55 & 8.55 \\
\hline 53.57 & 8.18 & 53.98 & 8.26 & 53.52 & 8.55 \\
\hline 78.93 & 8.16 & 79.35 & 8.25 & 78.90 & 8.52 \\
\hline 124.6 & 8.12 & 125.0 & 8.22 & 124.5 & 8.48 \\
\hline 147.5 & 8.20 & 147.9 & 8.27 & 147.4 & 8.55 \\
\hline 173.9 & 8.14 & 174.3 & 8.23 & 173.8 & 8.49 \\
\hline \multicolumn{6}{|c|}{ Initial $\mathrm{pH}=10.00$} \\
\hline .00 & 10.00 & .00 & 10.00 & .00 & 10.00 \\
\hline .75 & 10.03 & .67 & 10.02 & .57 & 10.07 \\
\hline 27.12 & 9.69 & 27.02 & 9.70 & 26.97 & 9.74 \\
\hline 49.10 & 9.69 & 49.00 & 9.71 & 48.90 & 9.75 \\
\hline 74.48 & 9.63 & 74.38 & 9.65 & 74.28 & 9.71 \\
\hline 120.1 & 9.61 & 120.0 & 9.62 & 119.9 & 9.67 \\
\hline 142.8 & 9.65 & 142.7 & 9.68 & 142.5 & 9.75 \\
\hline 169.4 & 9.60 & 169.3 & 9.62 & 169.2 & 9.66 \\
\hline
\end{tabular}


Table 42.-Variation of pH with reaction time for different combinations of Initial pH and Initlal free-chlorine concentration, Mlssissippl River at Baton Rouge, La.,

September 26, 1991

[CL, initial free-chlorine concentration; $\mathrm{mg} / \mathrm{L}$, milligram per liter]

\begin{tabular}{|c|c|c|c|c|c|}
\hline \multicolumn{2}{|c|}{$C L=15.0 \mathrm{mg} / \mathrm{L}$} & \multicolumn{2}{|c|}{$C L=30.0 \mathrm{mg} / \mathrm{L}$} & \multicolumn{2}{|c|}{$\mathrm{CL}=50.0 \mathrm{mg} / \mathrm{L}$} \\
\hline $\begin{array}{c}\text { Reaction } \\
\text { time } \\
\text { (hours) }\end{array}$ & pH & $\begin{array}{c}\text { Reaction } \\
\text { time } \\
\text { (hours) }\end{array}$ & pH & $\begin{array}{c}\text { Reaction } \\
\text { tlme } \\
\text { (hours) }\end{array}$ & pH \\
\hline \multicolumn{6}{|c|}{ Initial pH $=\mathbf{5 . 5 0}$} \\
\hline 0.00 & 5.50 & 0.00 & 5.50 & 0.00 & 5.50 \\
\hline 4.10 & 6.50 & 4.00 & 6.71 & 3.88 & 7.02 \\
\hline 29.45 & 6.56 & 29.35 & 6.74 & 29.23 & 7.02 \\
\hline 55.72 & 6.59 & 55.63 & 6.78 & 55.55 & 7.09 \\
\hline 79.53 & 6.65 & 79.43 & 6.80 & 79.32 & 7.18 \\
\hline 103.7 & 6.71 & 103.6 & 6.86 & 103.5 & 7.30 \\
\hline 128.1 & 6.79 & 128.0 & 6.90 & 127.9 & 7.33 \\
\hline 171.8 & 6.90 & 171.7 & 6.95 & 171.6 & 7.41 \\
\hline \multicolumn{6}{|c|}{ Initial $\mathrm{pH}=\mathbf{7 . 5 0}$} \\
\hline .00 & 7.50 & .00 & 7.50 & .00 & 7.50 \\
\hline 3.32 & 8.20 & 3.07 & 8.33 & 3.30 & 8.62 \\
\hline 28.65 & 8.16 & 28.37 & 8.27 & 28.58 & 8.57 \\
\hline 54.97 & 8.14 & 54.68 & 8.26 & 54.90 & 8.57 \\
\hline 78.73 & 8.14 & 78.45 & 8.26 & 78.68 & 8.55 \\
\hline 102.9 & 8.19 & 102.6 & 8.27 & 102.8 & 8.53 \\
\hline 127.3 & 8.19 & 127.0 & 8.27 & 127.2 & 8.49 \\
\hline 171.0 & 8.22 & 170.7 & 8.31 & 171.0 & 8.50 \\
\hline \multicolumn{6}{|c|}{ Initial $\mathrm{pH}=10.00$} \\
\hline .00 & 10.00 & .00 & 10.00 & .00 & 10.00 \\
\hline 1.63 & 9.97 & 1.62 & 9.95 & 1.52 & 9.99 \\
\hline 26.90 & 9.70 & 26.80 & 9.70 & 26.70 & 9.76 \\
\hline 53.27 & 9.66 & 53.17 & 9.67 & 53.07 & 9.73 \\
\hline 77.00 & 9.63 & 76.90 & 9.65 & 76.82 & 9.71 \\
\hline 101.2 & 9.60 & 101.1 & 9.61 & 101.0 & 9.68 \\
\hline 125.6 & 9.57 & 125.5 & 9.59 & 125.4 & 9.66 \\
\hline 169.3 & 9.56 & 169.2 & 9.61 & 169.1 & 9.67 \\
\hline
\end{tabular}


Table 43.-Varlation of $\mathrm{pH}$ with reaction time for different comblnatlons of Inltial pH and Initial free-chlorlne concentration, New Orleans, La., September 25, 1991

[CL, initial free-chlorine concentration; $\mathrm{mg} / \mathrm{L}$, milligram per liter]

\begin{tabular}{|c|c|c|c|c|c|}
\hline \multicolumn{2}{|c|}{$C L=15.0 \mathrm{mg} / \mathrm{L}$} & \multicolumn{2}{|c|}{$\mathrm{CL}=30.0 \mathrm{mg} / \mathrm{L}$} & \multicolumn{2}{|c|}{$\mathrm{CL}=50.0 \mathrm{mg} / \mathrm{L}$} \\
\hline $\begin{array}{l}\text { Reaction } \\
\text { time } \\
\text { (hours) }\end{array}$ & pH & $\begin{array}{c}\text { Reaction } \\
\text { time } \\
\text { (hours) }\end{array}$ & pH & $\begin{array}{c}\text { Reactlon } \\
\text { time } \\
\text { (hours) }\end{array}$ & pH \\
\hline \multicolumn{6}{|c|}{ Initial pH $=\mathbf{5 . 5 0}$} \\
\hline 0.00 & 5.50 & 0.00 & 5.50 & 0.00 & 5.50 \\
\hline 2.07 & 6.44 & 2.02 & 6.60 & 1.93 & 6.94 \\
\hline 31.12 & 6.49 & 31.03 & 6.67 & 30.93 & 6.94 \\
\hline 54.35 & 6.52 & 54.27 & 6.70 & 54.17 & 6.96 \\
\hline 78.87 & 6.66 & 78.80 & 6.81 & 78.70 & 7.02 \\
\hline 124.4 & 6.72 & 124.4 & 6.88 & 124.3 & 7.04 \\
\hline 170.6 & 6.85 & 170.6 & 6.94 & 170.4 & 7.09 \\
\hline \multicolumn{6}{|c|}{ Initial $\mathbf{p H}=7.50$} \\
\hline .00 & 7.50 & .00 & 7.50 & .00 & 7.50 \\
\hline 3.05 & 8.28 & 3.38 & 8.36 & 3.05 & 8.65 \\
\hline 32.12 & 8.18 & 32.43 & 8.28 & 32.05 & 8.57 \\
\hline 55.28 & 8.15 & 55.60 & 8.23 & 55.22 & 8.52 \\
\hline 79.82 & 8.16 & 80.28 & 8.23 & 79.90 & 8.52 \\
\hline 125.4 & 8.17 & 125.7 & 8.22 & 125.3 & 8.51 \\
\hline 171.6 & 8.19 & 171.9 & 8.23 & 171.5 & 8.51 \\
\hline \multicolumn{6}{|c|}{ Initial $\mathrm{pH}=10.00$} \\
\hline .00 & 10.00 & .00 & 10.00 & .00 & 10.00 \\
\hline .92 & 10.03 & .83 & 10.05 & .75 & 10.06 \\
\hline 29.92 & 9.74 & 29.82 & 9.77 & 29.72 & 9.80 \\
\hline 53.08 & 9.65 & 53.00 & 9.69 & 52.92 & 9.71 \\
\hline 77.77 & 9.67 & 77.67 & 9.70 & 77.57 & 9.72 \\
\hline 123.2 & 9.61 & 123.1 & 9.66 & 123.0 & 9.69 \\
\hline 169.4 & 9.57 & 169.3 & 9.66 & 169.2 & 9.68 \\
\hline
\end{tabular}




\section{Values of $\mathrm{pH}$ at the End of the Trihalomethane Formation-Potential Experiments}

Values of $\mathrm{pH}$ at the end of the trihalomethane formation-potential experiments for each of the different combinations of initial $\mathrm{pH}$ and initial free-chlorine concentration are listed in table 44. These $\mathrm{pH}$ values differ somewhat from the $\mathrm{pH}$ values in tables $30-43$ because they were determined on samples that had been quenched with a sodium thiosulfate solution and extracted with pentane, followed by evaporation of the pentane. Most of the small differences are believed to be from the addition of the sodium thiosulfate, which generally increased the $\mathrm{pH}$ values of the 5.50 and 7.50 samples and had little or no effect on the $\mathrm{pH}$ values of the 10.00 samples.

\section{Dissolved Organic-Carbon and Bromide Concentrations and Specific Conductances}

DOC and bromide concentrations and specific conductances for each of the water samples are listed in table 45. Replicate analyses were done for DOC for all samples. Coefficients of variation of the replicate analyses ranged from \pm 0.00 to \pm 1.30 and averaged \pm 0.43 percent for 14 comparisons. Bromide concentrations ranged from less than the detection limit of $0.005 \mathrm{mg} / \mathrm{L}$ for the Minneapolis, LaCrosse, and Dubuque water samples to $0.085 \mathrm{mg} / \mathrm{L}$ for the Missouri River water sample. An interference problem prevented the determiration of the bromide concentrations for the Davenport and Quincy water samples.

\section{Ulitravlolet Absorbances}

Ultraviolet absorbances of the natural water samples and the water samples adjusted to $\mathrm{pH}$ values of 5.50,7.50, and 10.00 were measured at $254,280,330$, and $400 \mathrm{~nm}$, and the results are listed in table 46. The $\mathrm{pH}$ value of the natural water sample for each sample location is the average of the $\mathrm{pH}$ values for the three volumes of water used for the formation-potential experiments and the one volume used for the preparation of the THM standards. Meaningful absorbance measurements were not obtained for the $\mathrm{pH}=10.00$ condition for the Minneapolis and LaCrosse water samples because a slight precipitate formed, resulting in a cloudy solution when the natural water $\mathrm{pH}$ was adjusted to 10.00. Much less intense precipitates were observed in the Dubuque, Davenport, Quincy, Missouri River, St. Louis, and Cairo water samples for the $\mathrm{pH}=10.00$ condition. The extent to which the UV absorbances of these samples were affected by this precipitate is not known. 
Table 44.-Values of $\mathrm{pH}$ at the end of the trihalomethane formation-potential experlments

[CL, initial free-chlorine concentration; $\mathrm{mg} / \mathrm{L}$, milligram per liter ]

\begin{tabular}{|c|c|c|c|c|}
\hline \multirow[t]{2}{*}{ General location } & \multirow{2}{*}{$\begin{array}{c}\text { Inltial } \\
\text { pH }\end{array}$} & \multicolumn{3}{|c|}{$\frac{C L}{(m g / L)}$} \\
\hline & & 15.0 & 30.0 & 50.0 \\
\hline \multirow[t]{3}{*}{ Minneapolis, Minn. } & 5.50 & 6.76 & 6.82 & 7.08 \\
\hline & 7.50 & 8.28 & 8.21 & 8.57 \\
\hline & 10.00 & 9.47 & 9.58 & 9.68 \\
\hline \multirow[t]{3}{*}{ LaCrosse, Wis. } & 5.50 & 6.78 & 6.87 & 7.19 \\
\hline & 7.50 & 8.17 & 8.21 & 8.73 \\
\hline & 10.00 & 9.59 & 9.60 & 9.70 \\
\hline \multirow[t]{3}{*}{ Dubuque, Iowa } & 5.50 & 7.12 & 7.25 & 7.55 \\
\hline & 7.50 & 8.28 & 8.41 & 8.84 \\
\hline & 10.00 & 9.48 & 9.58 & 9.69 \\
\hline \multirow[t]{3}{*}{ Davenport, Iowa } & 5.50 & 6.64 & 6.83 & 7.18 \\
\hline & 7.50 & 8.17 & 8.36 & 8.83 \\
\hline & 10.00 & 9.51 & 9.62 & 9.72 \\
\hline \multirow[t]{3}{*}{ Quincy, Ill. } & 5.50 & 6.88 & 7.01 & 7.28 \\
\hline & 7.50 & 8.26 & 8.39 & 8.84 \\
\hline & 10.00 & 9.49 & 9.59 & 9.50 \\
\hline \multirow[t]{3}{*}{ Missouri River near confluence with Mississippi River } & 5.50 & 7.02 & 7.19 & 7.54 \\
\hline & 7.50 & 8.40 & 8.62 & 8.93 \\
\hline & 10.00 & 9.25 & 9.35 & 9.47 \\
\hline \multirow[t]{3}{*}{ St. Louis, Mo. } & 5.50 & 6.81 & 6.96 & 7.27 \\
\hline & 7.50 & 8.22 & 8.50 & 8.89 \\
\hline & 10.00 & 9.42 & 9.58 & 9.71 \\
\hline \multirow[t]{3}{*}{ Cairo, Ill. } & 5.50 & 6.72 & 7.04 & 7.15 \\
\hline & 7.50 & 8.02 & 8.28 & 8.69 \\
\hline & 10.00 & 9.51 & 9.60 & 9.72 \\
\hline \multirow[t]{3}{*}{ Ohio River near confluence with Mississippi River } & 5.50 & 6.99 & 7.37 & 8.27 \\
\hline & 7.50 & 8.41 & 8.83 & 9.19 \\
\hline & 10.00 & 9.39 & 9.63 & 9.85 \\
\hline \multirow[t]{3}{*}{ Memphis, Tenn. } & 5.50 & 6.92 & 7.12 & 7.50 \\
\hline & 7.50 & 8.26 & 8.56 & 8.98 \\
\hline & 10.00 & 9.50 & 9.65 & 9.81 \\
\hline \multirow[t]{3}{*}{ Greenville, Miss. } & 5.50 & 7.33 & 7.41 & 7.76 \\
\hline & 7.50 & 8.48 & 8.69 & 9.04 \\
\hline & 10.00 & 9.44 & 9.55 & 9.61 \\
\hline
\end{tabular}


Table 44.-Vaiues of $\mathrm{pH}$ at the end of the trihaiomethane formation-potential experiments -Continued

\begin{tabular}{lccccc}
\hline Generai iocation & Initiai & \multicolumn{3}{c}{ CL } \\
& pH & & (mg/L) & \\
\cline { 5 - 6 } & & & $\mathbf{1 5 . 0}$ & $\mathbf{3 0 . 0}$ & $\mathbf{5 0 . 0}$ \\
\hline Natchez, Miss. & 7.50 & 7.15 & 7.35 & 7.72 \\
& 10.00 & 9.49 & 8.84 & 9.15 \\
& & & 9.69 & 9.80 \\
Baton Rouge, La. & 5.50 & 6.93 & 7.21 & 7.65 \\
& 7.50 & 8.49 & 8.88 & 9.25 \\
& 10.00 & 9.66 & 9.78 & 9.90 \\
New Orleans, La. & & & & \\
& 5.50 & 7.03 & 7.26 & 7.58 \\
& 7.50 & 8.44 & 8.76 & 9.09 \\
& 10.00 & 9.51 & 9.62 & 9.72 \\
\hline
\end{tabular}

Tabie 45.-Dissoived organic-carbon and bromide concentrations and specific conductances of the water sampies

[DOC, dissolved organic carbon; $\mathrm{Br}$, bromide; Rep 1, replicate number 1; Rep 2, replicate number $2 ; \mathrm{mg} / \mathrm{L}$, milligram per liter; $\mu \mathrm{S} / \mathrm{cm}$, microsiemens per centimeter at 25 degrees Celsius; ND, not detected; IF, interference problem]

\begin{tabular}{|c|c|c|c|c|}
\hline \multirow{3}{*}{ Generai iocation } & \multicolumn{3}{|c|}{ Concentration } & \multirow{3}{*}{$\begin{array}{c}\text { Specific } \\
\text { conductance } \\
(\mu \mathrm{S} / \mathrm{cm})\end{array}$} \\
\hline & \multicolumn{2}{|c|}{$\overline{\text { DOC }}$} & \multirow{2}{*}{$\underset{(\mathrm{mg} / \mathrm{L})}{\mathrm{Br}}$} & \\
\hline & $\begin{array}{l}\text { Rep 1 } \\
\text { (mg/l) }\end{array}$ & $\begin{array}{c}\text { Rep 2 } \\
\text { (mg/L) }\end{array}$ & & \\
\hline Minneapolis, Minn. & 11 & 11 & ND & 473 \\
\hline LaCrosse, Wis. & 8.9 & 9.1 & ND & 447 \\
\hline Dubuque, Iowa & 7.6 & 7.7 & ND & 395 \\
\hline Davenport, Iowa & 7.7 & 7.6 & IF & 404 \\
\hline Quincy, Ill. & 7.2 & 7.1 & IF & 469 \\
\hline Missouri River ${ }^{1}$ & 2.7 & 2.7 & .085 & 719 \\
\hline St. Louis, Mo. & 4.0 & 4.0 & .044 & 622 \\
\hline Cairo, Ill. & 4.8 & 4.8 & .031 & 564 \\
\hline Ohio River ${ }^{1}$ & 2.2 & 2.2 & .042 & 280 \\
\hline Memphis, Tenn. & 3.8 & 3.8 & .040 & 463 \\
\hline Greenville, Miss. & 3.8 & 3.9 & .040 & 464 \\
\hline Natchez, Miss. & 3.3 & 3.3 & .039 & 434 \\
\hline Baton Rouge, La. & 3.1 & 3.2 & .044 & 433 \\
\hline New Orleans, La. & 3.5 & 3.5 & .044 & 457 \\
\hline
\end{tabular}

${ }^{1}$ Sampling site 1.6 kilometers upstream from confluence with Mississippi River. 
Table 46.-Ultraviolet absorbances of the water samples

[nm, nanometer; PP, precipitate problem]

\begin{tabular}{|c|c|c|c|c|c|}
\hline \multirow{2}{*}{ General location } & \multirow{2}{*}{ pH } & \multicolumn{4}{|c|}{$\begin{array}{c}\text { Ultraviolet absorbance, in absorbance units, } \\
\text { at wavelengths }\end{array}$} \\
\hline & & $\begin{array}{c}254 \\
(\mathrm{~nm})\end{array}$ & $\begin{array}{c}280 \\
(\mathrm{~nm})\end{array}$ & $\begin{array}{c}330 \\
(\mathrm{~nm})\end{array}$ & $\begin{array}{c}400 \\
(\mathrm{~nm})\end{array}$ \\
\hline \multirow[t]{4}{*}{ Minneapolis, Minn. } & 8.15 & 1.743 & 1.263 & 0.545 & 0.155 \\
\hline & 5.50 & 1.737 & 1.249 & .519 & .135 \\
\hline & 7.50 & 1.733 & 1.255 & .536 & .150 \\
\hline & 10.00 & PP & PP & PP & PP \\
\hline \multirow[t]{4}{*}{ LaCrosse, Wis. } & 8.08 & 1.550 & 1.135 & .508 & .146 \\
\hline & 5.50 & 1.540 & 1.117 & .481 & .126 \\
\hline & 7.50 & 1.548 & 1.127 & .499 & .141 \\
\hline & 10.00 & PP & PP & $\mathbf{P P}$ & PP \\
\hline \multirow[t]{4}{*}{ Dubuque, Iowa } & 8.01 & 1.307 & .950 & .409 & .117 \\
\hline & 5.50 & 1.300 & .938 & .392 & .103 \\
\hline & 7.50 & 1.304 & .947 & .406 & .114 \\
\hline & 10.00 & 1.355 & .994 & .472 & .183 \\
\hline \multirow[t]{4}{*}{ Davenport, Iowa } & 8.03 & 1.222 & .882 & .370 & .102 \\
\hline & 5.50 & 1.217 & .874 & .358 & .090 \\
\hline & 7.50 & 1.219 & .879 & .367 & .100 \\
\hline & 10.00 & 1.225 & .886 & .388 & .118 \\
\hline \multirow[t]{4}{*}{ Quincy, Ill. } & 8.19 & 1.135 & .817 & .338 & .093 \\
\hline & 5.50 & 1.128 & .811 & .324 & .084 \\
\hline & 7.50 & 1.126 & .813 & .333 & .090 \\
\hline & 10.00 & 1.094 & .790 & .332 & .095 \\
\hline \multirow{4}{*}{$\begin{array}{l}\text { Missouri River near conflu- } \\
\text { ence with Mississippi River }\end{array}$} & 8.34 & .339 & .237 & .091 & .025 \\
\hline & 5.50 & .336 & .238 & .089 & .024 \\
\hline & 7.50 & .338 & .239 & .091 & .025 \\
\hline & 10.00 & .324 & .227 & .088 & .028 \\
\hline \multirow[t]{4}{*}{ St. Louis, Mo. } & 8.27 & .598 & .427 & .170 & .048 \\
\hline & 5.50 & .595 & .422 & .163 & .043 \\
\hline & 7.50 & .596 & .426 & .167 & .046 \\
\hline & 10.00 & .565 & .419 & .174 & .063 \\
\hline
\end{tabular}


Table 46.-Ultraviolet absorbances of the water samples -Continued

\begin{tabular}{|c|c|c|c|c|c|}
\hline \multirow{2}{*}{ General location } & \multirow{2}{*}{ pH } & \multicolumn{4}{|c|}{$\begin{array}{l}\text { Ultraviolet absorbance, in absorbance units, } \\
\text { at wavelengths }\end{array}$} \\
\hline & & $\begin{array}{c}254 \\
(\mathrm{~nm})\end{array}$ & $\begin{array}{l}280 \\
(\mathrm{~nm})\end{array}$ & $\begin{array}{l}330 \\
(\mathrm{~nm})\end{array}$ & $\begin{array}{l}400 \\
(\mathrm{~nm})\end{array}$ \\
\hline \multirow[t]{4}{*}{ Cairo, Ill. } & 8.11 & .756 & .544 & .216 & .060 \\
\hline & 5.50 & .752 & .537 & .209 & .054 \\
\hline & 7.50 & .753 & .540 & .214 & .058 \\
\hline & 10.00 & .714 & .519 & .213 & .063 \\
\hline Ohio River near confluence & 7.92 & .293 & .212 & .080 & .022 \\
\hline \multirow{3}{*}{ with Mississippi River } & 5.50 & .295 & .213 & .079 & .023 \\
\hline & 7.50 & .298 & .217 & .082 & .026 \\
\hline & 10.00 & .294 & .214 & .084 & .025 \\
\hline \multirow[t]{4}{*}{ Memphis, Tenn. } & 8.14 & .562 & .400 & .158 & .044 \\
\hline & 5.50 & .563 & .400 & .153 & .041 \\
\hline & 7.50 & .563 & .402 & .157 & .044 \\
\hline & 10.00 & .560 & .398 & .164 & .048 \\
\hline \multirow[t]{4}{*}{ Greenville, Miss. } & 8.06 & .501 & .357 & .139 & .039 \\
\hline & 5.50 & .497 & .354 & .135 & .037 \\
\hline & 7.50 & .500 & .357 & .139 & .038 \\
\hline & 10.00 & .488 & .349 & .137 & .039 \\
\hline \multirow[t]{4}{*}{ Natchez, Miss. } & 8.17 & .456 & .327 & .128 & .036 \\
\hline & 5.50 & .454 & .325 & .124 & .034 \\
\hline & 7.50 & .456 & .327 & .128 & .037 \\
\hline & 10.00 & .446 & .320 & .127 & .036 \\
\hline \multirow[t]{4}{*}{ Baton Rouge, La. } & 8.17 & .435 & .313 & .124 & .036 \\
\hline & 5.50 & .434 & .313 & .122 & .035 \\
\hline & 7.50 & .435 & .313 & .123 & .036 \\
\hline & 10.00 & .426 & .308 & .123 & .037 \\
\hline \multirow[t]{4}{*}{ New Orleans, La. } & 7.94 & .478 & .339 & .135 & .038 \\
\hline & 5.50 & .471 & .335 & .131 & .036 \\
\hline & 7.50 & .476 & .340 & .135 & .038 \\
\hline & 10.00 & .486 & .350 & .145 & .045 \\
\hline
\end{tabular}




\section{Error Analysis}

The coefficient of variation normalized with respect to the mean concentration and expressed as a percentage was used to indicate reproducibility between duplicate samples. This coefficient of variation was computed from

where

$$
C_{v}= \pm\left\{\left[C_{1}^{2}+C_{2}^{2}-\left(C_{1}+C_{2}\right)^{2} / 2\right] / 2\right\}^{0.5} 100 / C_{12}
$$

$\mathrm{C}_{\mathrm{v}}$ is the coefficient of variation;

$\mathrm{C}_{1}$ is concentration number 1 ;

$\mathrm{C}_{2}$ is concentration number 2; and

$\mathrm{C}_{12}$ is the mean of concentrations 1 and 2.

Values of the coefficients of variation for the analysis of duplicate THM samples and NPTOX samples are summarized in table 47. Minimum, maximum, and mean values of the coefficient of variation together with the number of pairs of values in each comparison are listed.

Table 47.-Minimum, maximum, and mean values of the coefficient of variation for analysis of duplicate trihalomethane and nonpurgeable total organic-halide samples

[THM, trihalomethane; NPTOX, nonpurgeable total organic halide]

\begin{tabular}{lcccc}
\hline \multirow{2}{*}{ Sample type } & Number of & \multicolumn{3}{c}{ Coefficient of variation, in percent } \\
\cline { 3 - 5 } & pairs & Minimum & Maximum & Mean \\
\hline THM & 126 & \pm 0.00 & \pm 6.08 & \pm 0.95 \\
NPTOX & 126 & \pm .00 & \pm 10.0 & \pm 2.45 \\
\hline
\end{tabular}




\section{REFERENCES CITED}

Bellar, T.A., Lichtenberg, J.J., and Kroner, R.C., 1974, The occurrence of organohalides in chlorinated drinking waters: Journal of American Water Works Association, v. 66, no. 12, p. 703-706.

Buffle, J., Deladoey, P., and Haerdi, W., 1978, The use of ultrafiltration for the separation and fractionation of organic ligands in fresh waters: Analytica Chimica Acta, v. 101, no. 2, p. 339-357.

Ceraso, Jane, 1987, Development of a surrogate parameter to determine the trihalomethane formation potential of two reservoirs: Journal of New England Water Works Association, v. 101 , no. 4 , p. $382-400$.

Chadick, P.A., and Amy, G.L., 1987, Coagulation and adsorption of humic substances-An analysis of surrogate parameters for predicting effects of trihalomethane formation potential: Environmental Technology Letters, v. 8, p. 261-268.

Edzwald, J.K., Becker, W.C., and Wattier, K.L., 1985, Surrogate parameters for monitoring organic matter and THM precursors: Journal of American Water Works Association, v. 77, no. 4, p. 122-132.

Federal Register, 1979, Analysis of trihalomethanes in drinking water, v. 44, no. 231, p. $68672-68690$.

Fishman, M.J., and Friedman, L.C., 1989, Methods for determination of inorganic substances in water and fluvial sediments: U.S. Geological Survey Techniques of Water-Resources Investigations Report, book 5, chap. A1, 545 p.

Fleischacker, S.J., and Randtke, S.J., 1983, Formation of organic chlorine in public water supplies: Journal of American Water Works Association, v. 75, no. 3, p. 132-138.

Johnson, J.D., and Jensen, J.N., 1986, THM and TOX formation-Routes, rates, and precursors: Journal of American Water Works Association, v. 78, no. 4, p. 156-162.

Moore, T.R., 1987, Assessment of a simple spectrophotometric method for the determination of dissolved organic carbon in freshwaters: New Zealand Journal of Marine and Freshwater Research, v. 21, no. 4, p. 585-589.

Oliver, B.G., and Thurman, E.M., 1981, Influence of aquatic humic substances on trihalomethane potential, in Proceedings, Fourth Conference on Water Chlorination, October 18-23, 1981, Environmental Impact and Health Effects: Ann Arbor, Mich., Ann Arbor Science Publishers, p. 231-241.

Rook, J.J., 1974, Formation of haloforms during chlorination of natural waters: Water Treatment and Examination, v. 23, no. 2, p. 234-243.

Wershaw, R.L., Fishman, M.J., Grabbe, R.R., and Lowe, L.E., 1987, Methods for the determination of organic substances in water and fluvial sediments: U.S. Geological Survey Techniques of Water-Resources Investigations, book 5, chap. A3, 80 p. 